\title{
Supporting Information for: Thermosensitive Hydration of Four Acrylamide-Based Polymers in Coil and Globule Conformations
}

Patrick K. Quoika' ${ }^{1}$, Maren Podewitz ${ }^{1}$, Yin Wang ${ }^{1}$, Anna S. Kamenik ${ }^{1}$, Johannes R. Loeffler ${ }^{1}$ and Klaus R. LiedI ${ }^{1 *}$

${ }^{1}$ Institute of General, Inorganic and Theoretical Chemistry, and Centre of Molecular Biosciences University of Innsbruck, A-6020 Innsbruck, Austria

E-mail: Klaus.liedl@uibk.ac.at 


$\begin{array}{ll}\text { Abbreviations and symbols } \\ \text { G } & \text { Coil } \\ \text { CGT } & \text { Globule } \\ \text { TSP } & \text { Coil-Globule transition } \\ \text { AAm } & \text { Thermosensitive polymers } \\ \text { NMAAm } & \text { poly-Acrylamide } \\ \text { NEAAm } & \text { poly-N-Methylacrylamide } \\ \text { NNIPAAm } & \text { poly-N-Isopropylacrylamide } \\ \mathrm{R}_{\mathrm{g}} & \text { Radius of gyration } \\ \mathrm{R}_{\mathrm{h}} & \text { Hydrodynamic radius } \\ \sigma & \text { Solvent accessible surface area } \\ \langle\tau\rangle & \text { Mean lifetime of hydrogen bonds } \\ \mathrm{v} & \text { Mean number of hydrogen bonds with water } \\ v_{\sigma} & \text { Mean number of hydrogen bonds with water per solvent accessible surface area } \\ \Delta_{C G} H & \text { Enthalpy difference with the CGT } \\ \Delta_{C G} H_{S o l} & \text { Enthalpy difference with the CGT originating from the solvent-solvent interactions } \\ \Delta_{C G} H_{P o l} & \text { Enthalpy difference with the CGT originating from the inner energy of the polymer } \\ \Delta_{C G} H_{P o l-S o l} & \text { Enthalpy difference with the CGT originating from the polymer-solvent interactions } \\ & \end{array}$




\section{SI figures}

Timeseries of Radius of Gyration, Solvent Accessible Surface Area and State
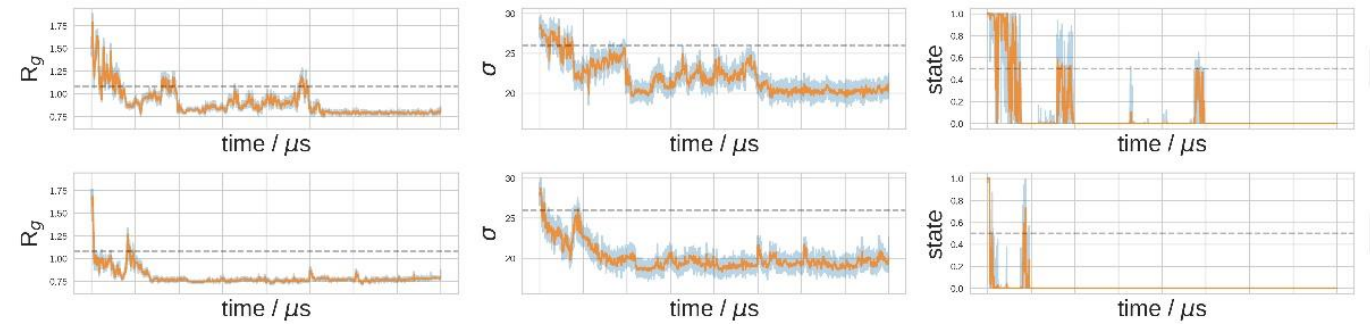

$\mathrm{T}=250 \mathrm{~K}$
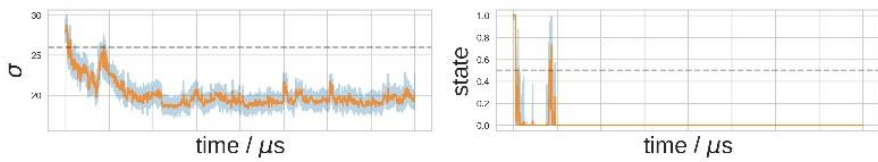

$\mathrm{T}=260 \mathrm{~K}$
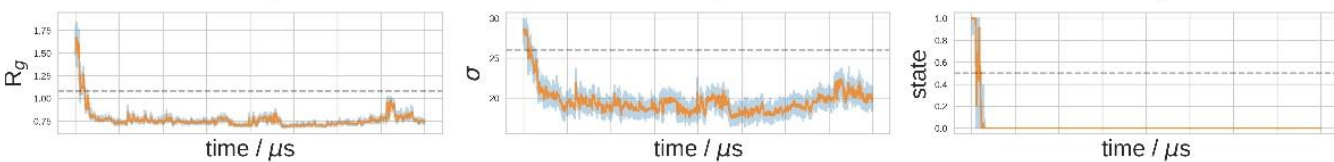

$\mathrm{T}=270 \mathrm{~K}$

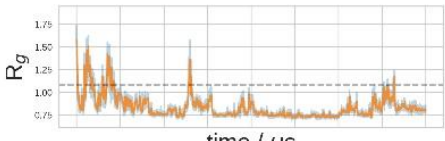

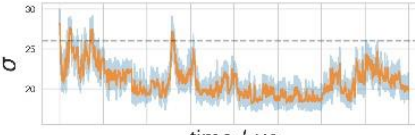

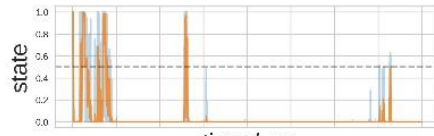

$\mathrm{T}=280 \mathrm{~K}$
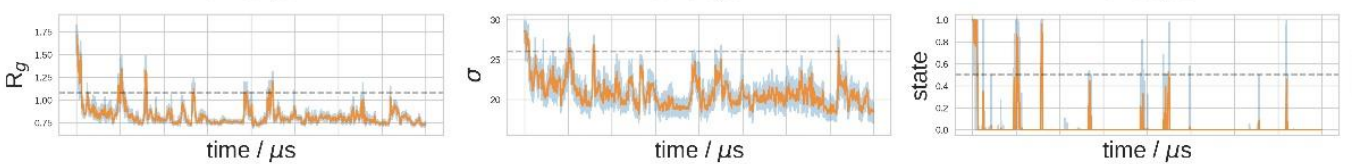

$\mathrm{T}=290 \mathrm{~K}$

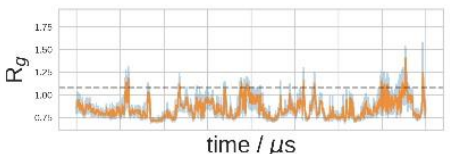

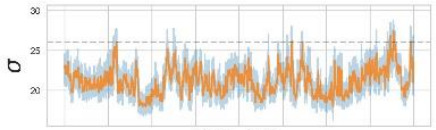

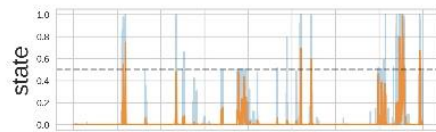

$\mathrm{T}=300 \mathrm{~K}$

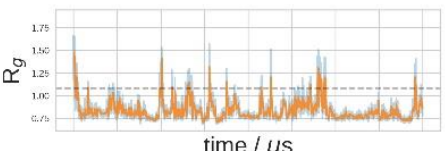

time $/ \mu \mathrm{s}$

time $/ \mu$ s
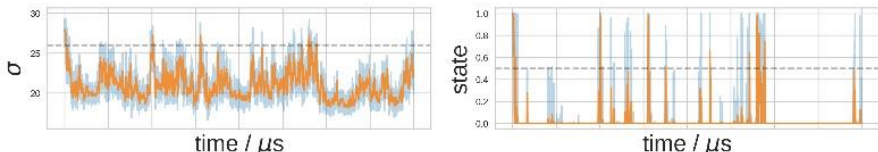

$\mathrm{T}=310 \mathrm{~K}$

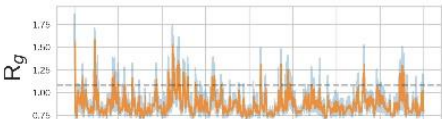

$0_{20}^{25}$ time $/ \mu \mathrm{s}$

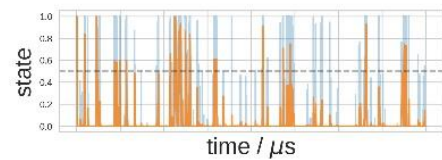

$\mathrm{T}=320 \mathrm{~K}$ time $/ \mu \mathrm{s}$
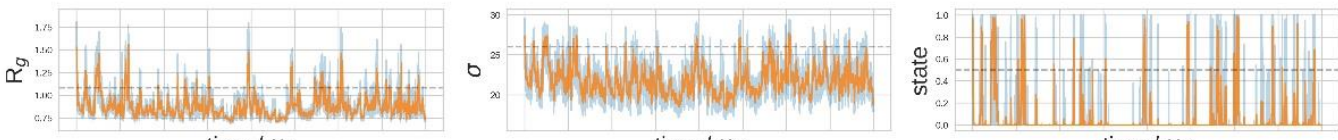

$\mathrm{T}=330 \mathrm{~K}$

time $/ \mu \mathrm{s}$
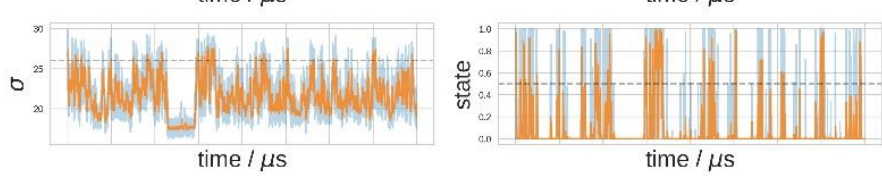

$\mathrm{T}=340 \mathrm{~K}$
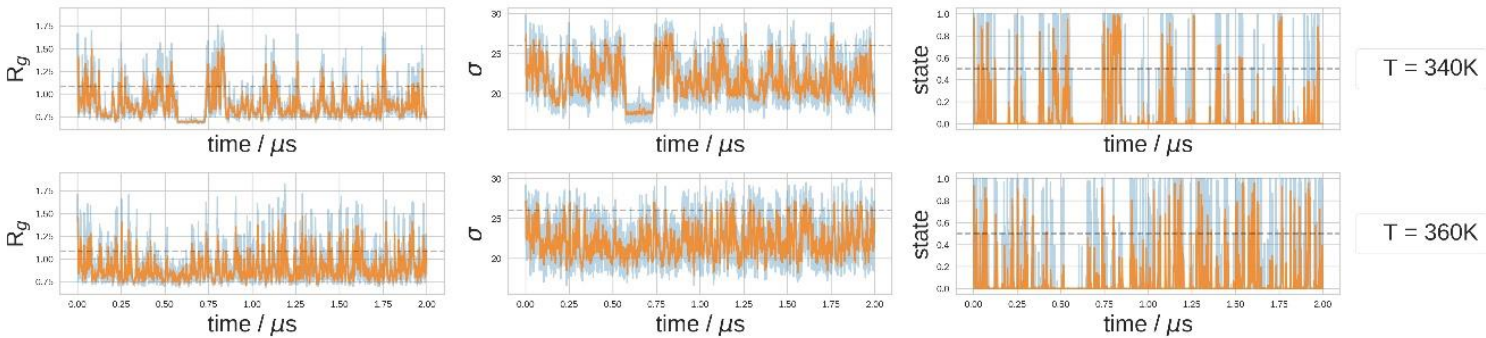

$\mathrm{T}=360 \mathrm{~K}$
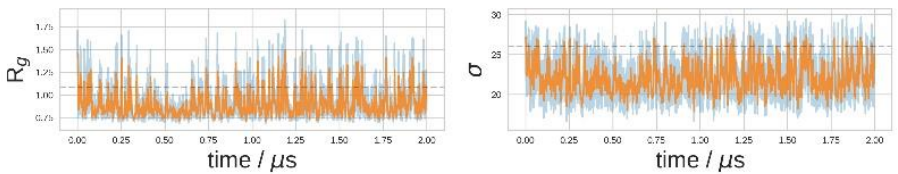

Figure S1 Timeseries of radius of gyration $\left(R_{g}\right)$, solvent accessible surface area $(\sigma)$ and conformational state derived from these two quantities for AAm 30mer at all simulated temperatures. Left column: $R_{g}$; middle column: $\sigma$; right column: state. In dashed lines, we show the state borders, defined from the $2 d$-histograms in the $R_{g}-\sigma$ plane. 

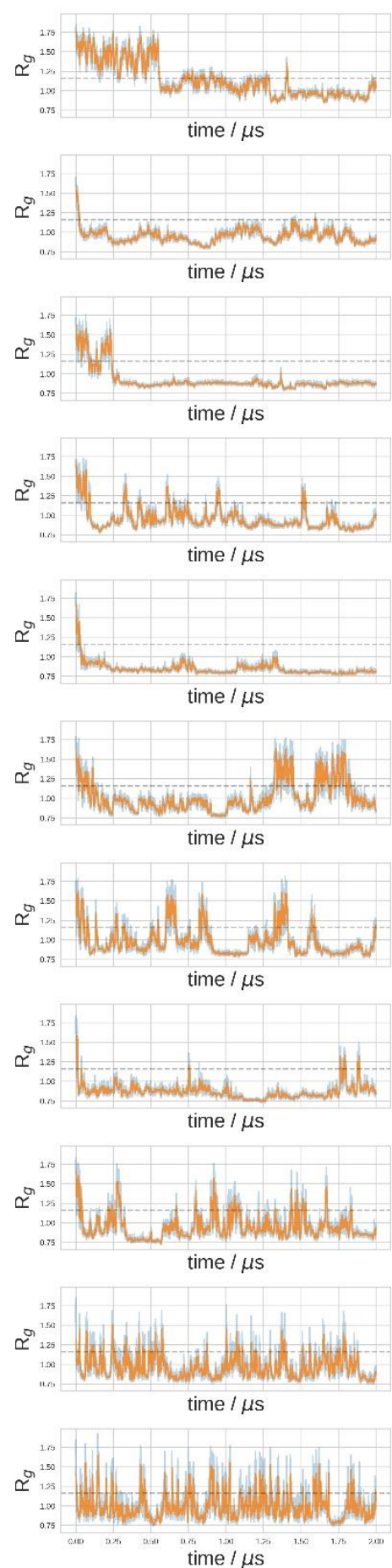
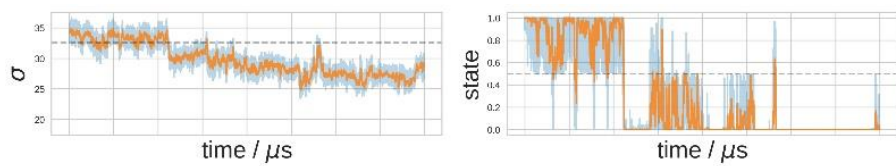

$\mathrm{T}=250 \mathrm{~K}$
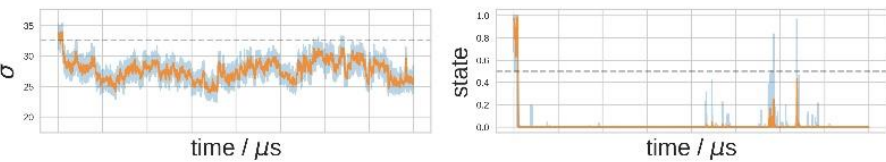

$T=260 \mathrm{~K}$
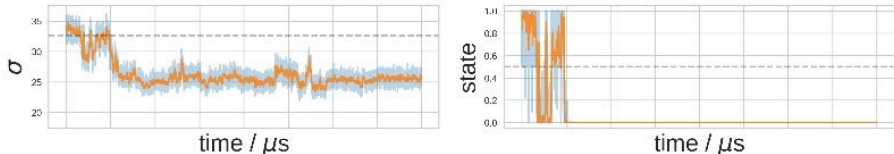

$\mathrm{T}=270 \mathrm{~K}$
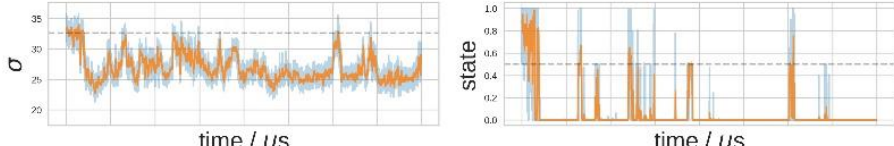

$\mathrm{T}=280 \mathrm{~K}$
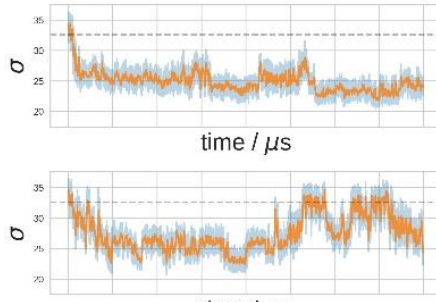

time $/ \mu$ s

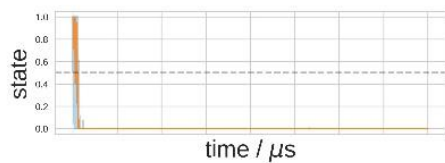

$\mathrm{T}=290 \mathrm{~K}$

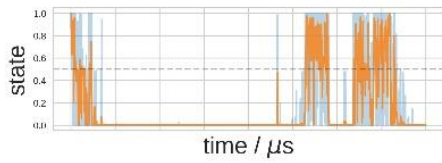

$\mathrm{T}=300 \mathrm{~K}$
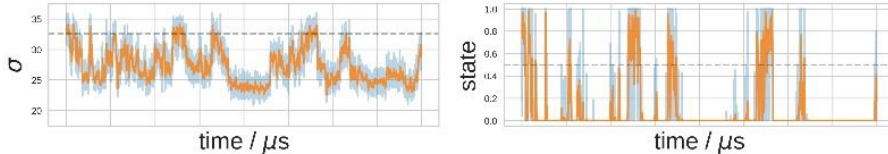

$\mathrm{T}=310 \mathrm{~K}$
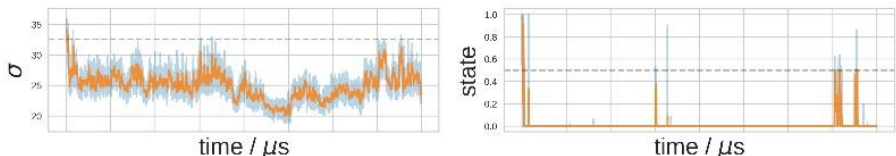

$\mathrm{T}=320 \mathrm{~K}$
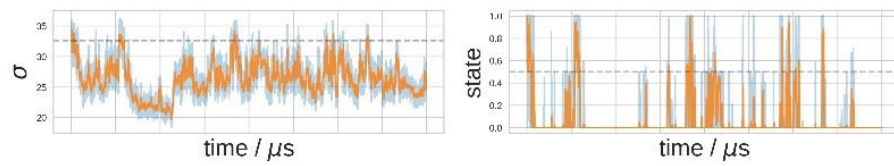

$\mathrm{T}=330 \mathrm{~K}$
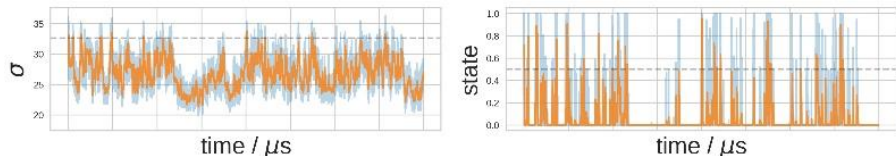

$\mathrm{T}=340 \mathrm{~K}$

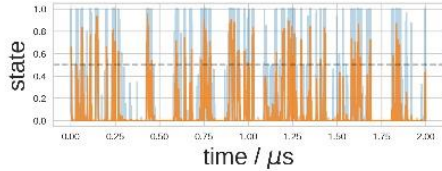

$\mathrm{T}=360 \mathrm{~K}$

Figure S2 Timeseries of radius of gyration $\left(R_{g}\right)$, solvent accessible surface area $(\sigma)$ and conformational state derived from these two quantities for NMAAm 30mer at all simulated temperatures. Left column: $R_{g}$; middle column: $\sigma$; right column: state. In dashed lines, we show the state borders, defined from the $2 d$-histograms in the $R_{g}$ - $\sigma$ plane. 

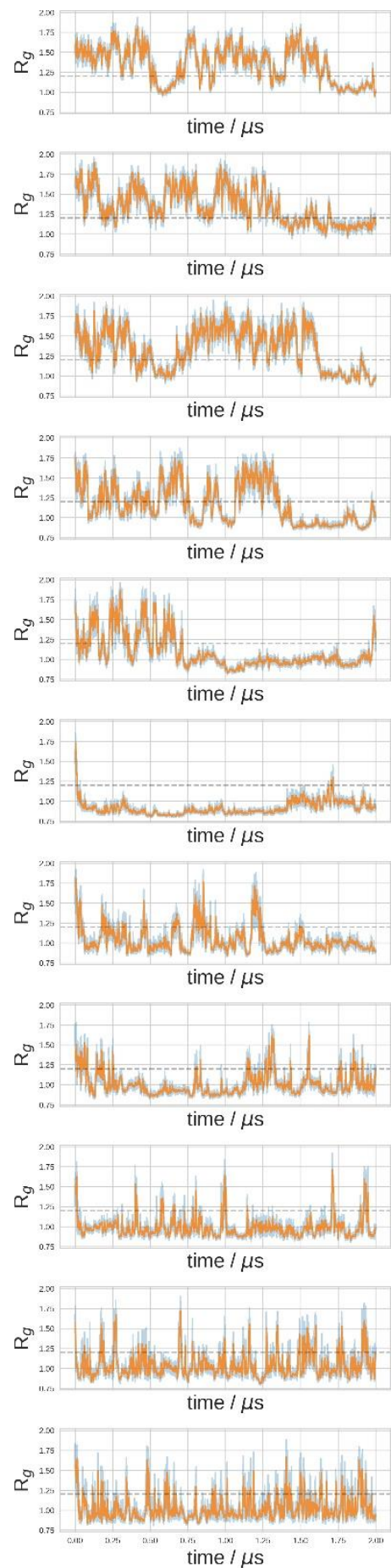
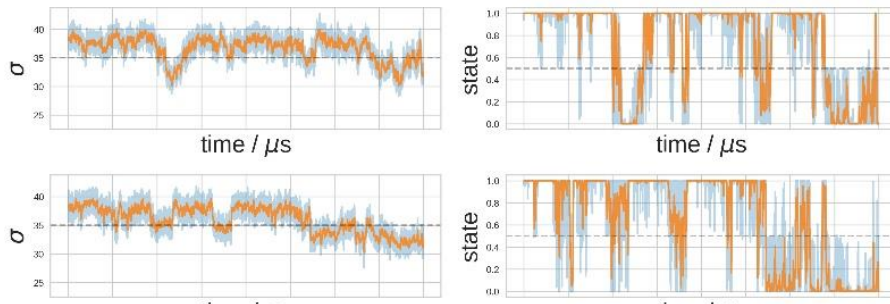

time $/ \mu \mathrm{s}$

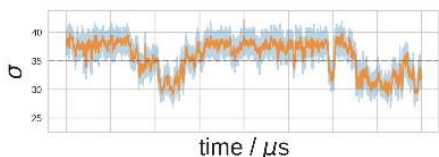

time $/ \mu \mathrm{s}$

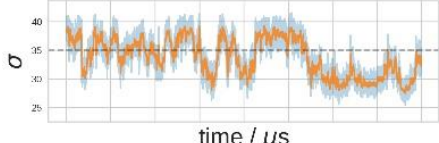

time $/ \mu \mathrm{s}$
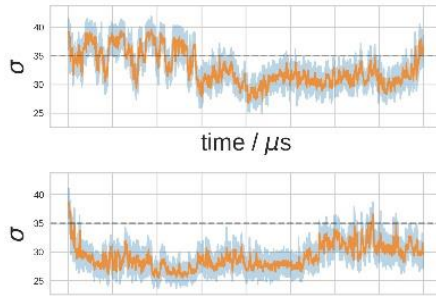

time $/ \mu \mathrm{s}$
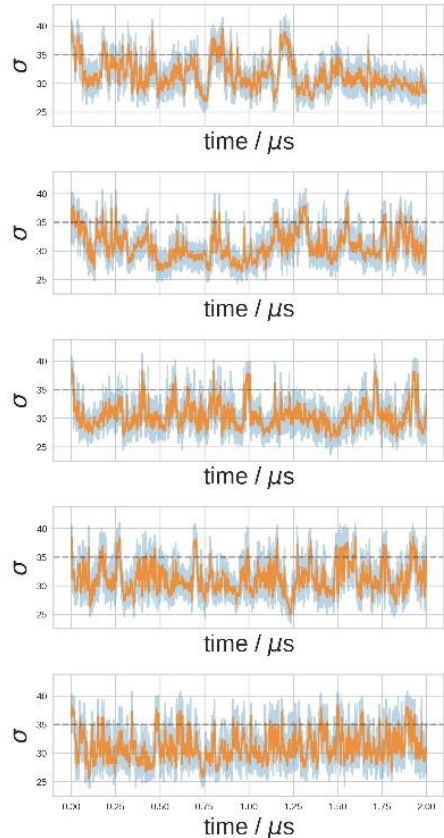

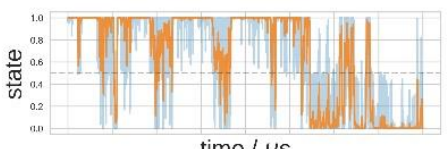

time $/ \mu$ s

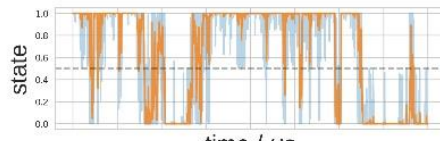

time $/ \mu \mathrm{s}$

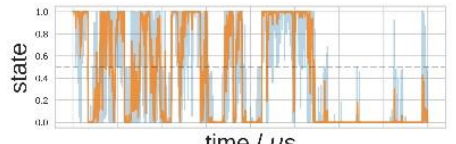

time $/ \mu \mathrm{s}$
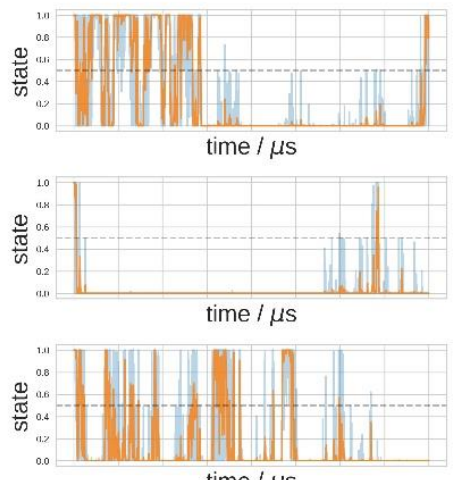

$\mathrm{T}=310 \mathrm{~K}$

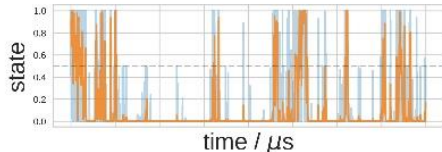

$\mathrm{T}=320 \mathrm{~K}$
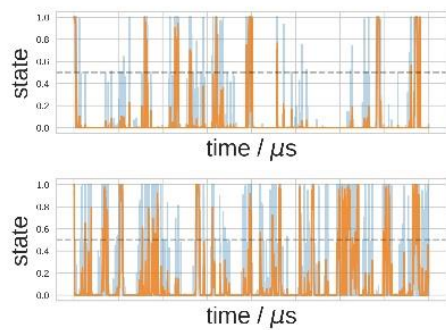

$\mathrm{T}=340 \mathrm{~K}$

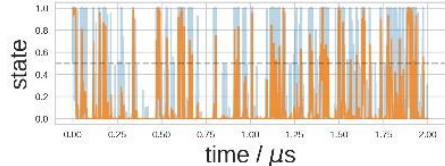

$\mathrm{T}=360 \mathrm{~K}$

Figure S3 Timeseries of radius of gyration $\left(R_{g}\right)$, solvent accessible surface area $(\sigma)$ and conformational state derived from these two quantities for NEAAm 30mer at all simulated temperatures. Left column: $R_{g}$; middle column: $\sigma$; right column: state. In dashed lines, we show the state borders, defined from the $2 d$-histograms in the $R_{g}-\sigma$ plane. 


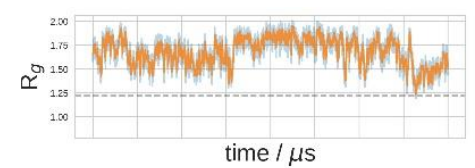

time $/ \mu$ s

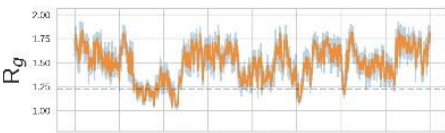

time $/ \mu \mathrm{s}$

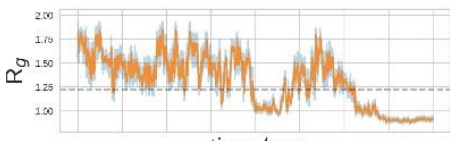

time $/ \mu$ s

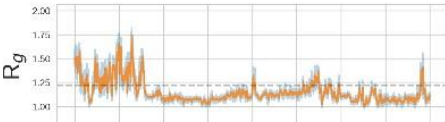

time $/ \mu \mathrm{s}$
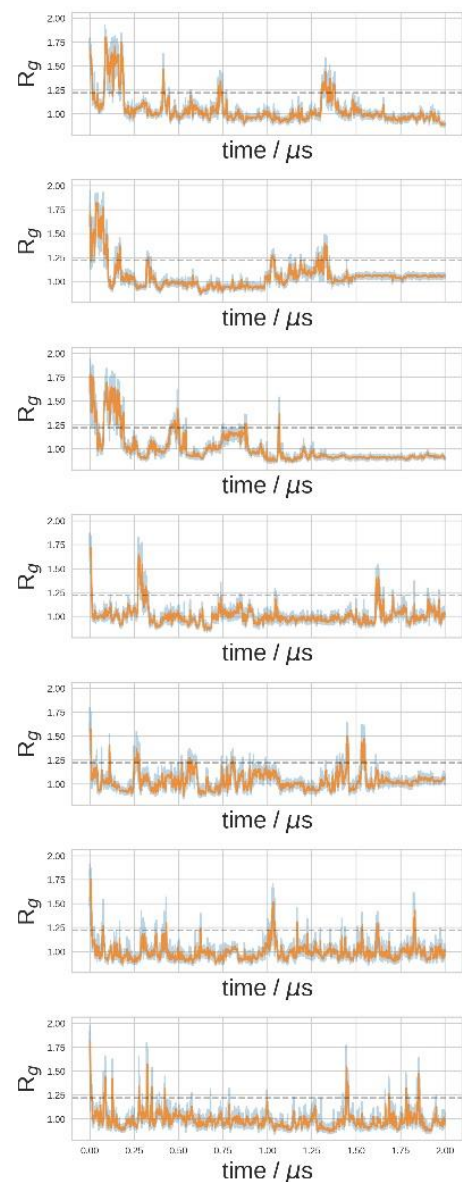

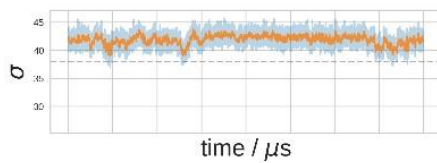

time $/ \mu$ s

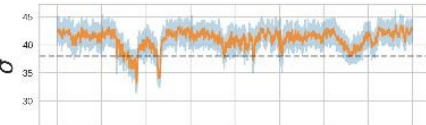

time $/ \mu$ s

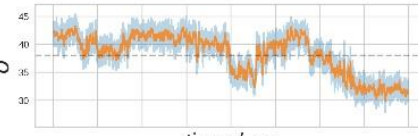

time $/ \mu \mathrm{s}$

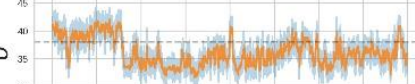

time $/ \mu$ s

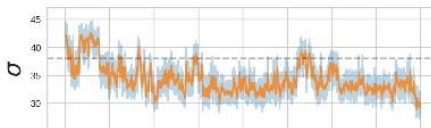

time $/ \mu$ s

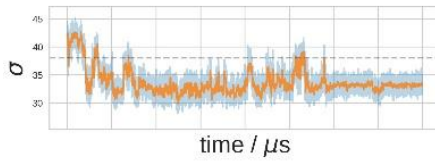

time $/ \mu$ s

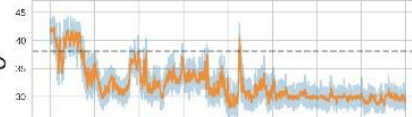

time $/ \mu$ s

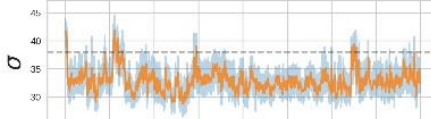

time $/ \mu$ s
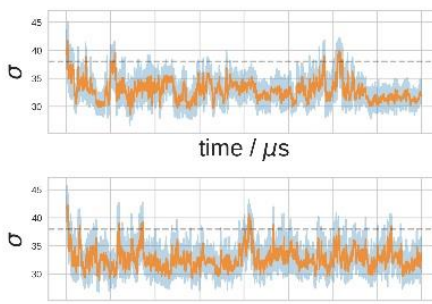

time $/ \mu$ s

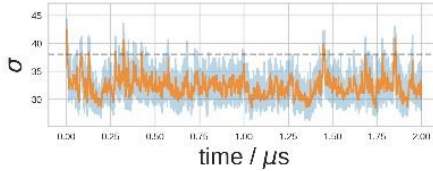

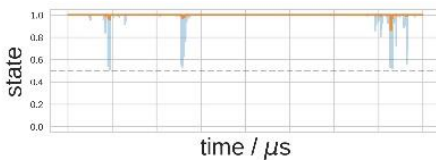

$\mathrm{T}=250 \mathrm{~K}$

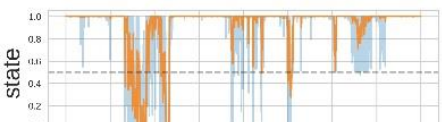

time $/ \mu$ s

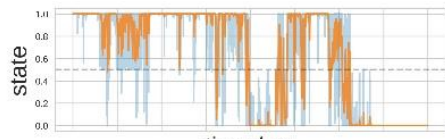

$\mathrm{T}=270 \mathrm{~K}$

time $/ \mu \mathrm{s}$

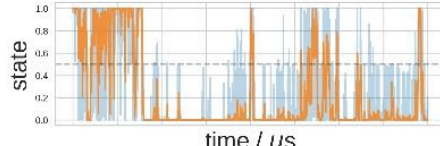

$\mathrm{T}=280 \mathrm{~K}$

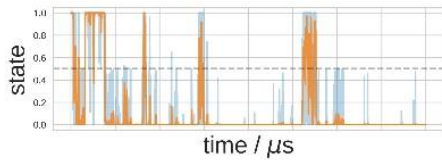

$\mathrm{T}=290 \mathrm{~K}$

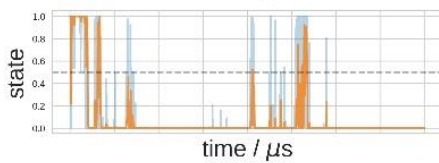

$\mathrm{T}=300 \mathrm{~K}$

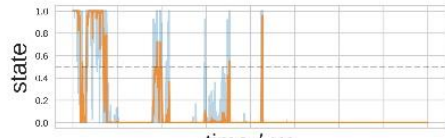

$\mathrm{T}=310 \mathrm{~K}$

time $/ \mu \mathrm{s}$

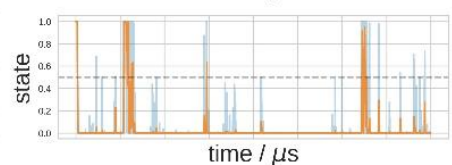

$\mathrm{T}=320 \mathrm{~K}$

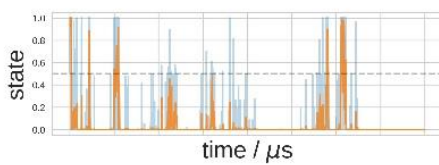

$\mathrm{T}=330 \mathrm{~K}$

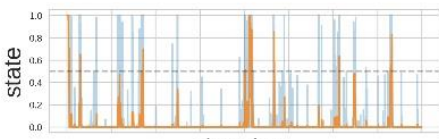

$\mathrm{T}=340 \mathrm{~K}$

time $/ \mu \mathrm{s}$

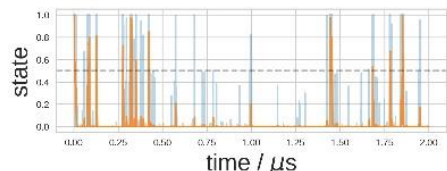

$\mathrm{T}=360 \mathrm{~K}$

Figure S4 Timeseries of radius of gyration $\left(R_{g}\right)$, solvent accessible surface area $(\sigma)$ and conformational state derived from these two quantities for NIPAAm 30mer at all simulated temperatures. Left column: $R_{g}$; middle column: $\sigma$; right column: state. In dashed lines, we show the state borders, defined from the $2 d$-histograms in the $R_{g}$ - $\sigma$ plane. 

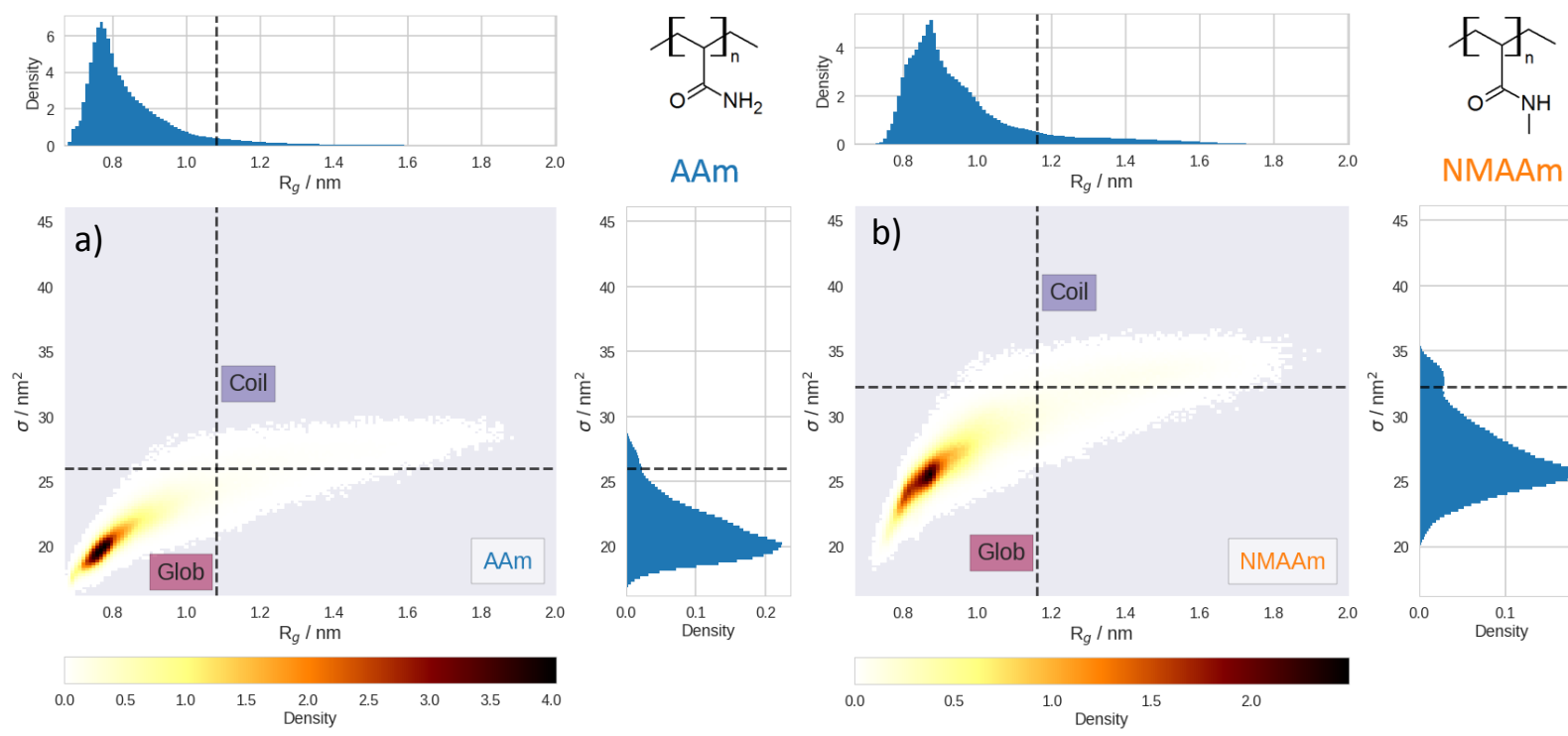

NMAAm
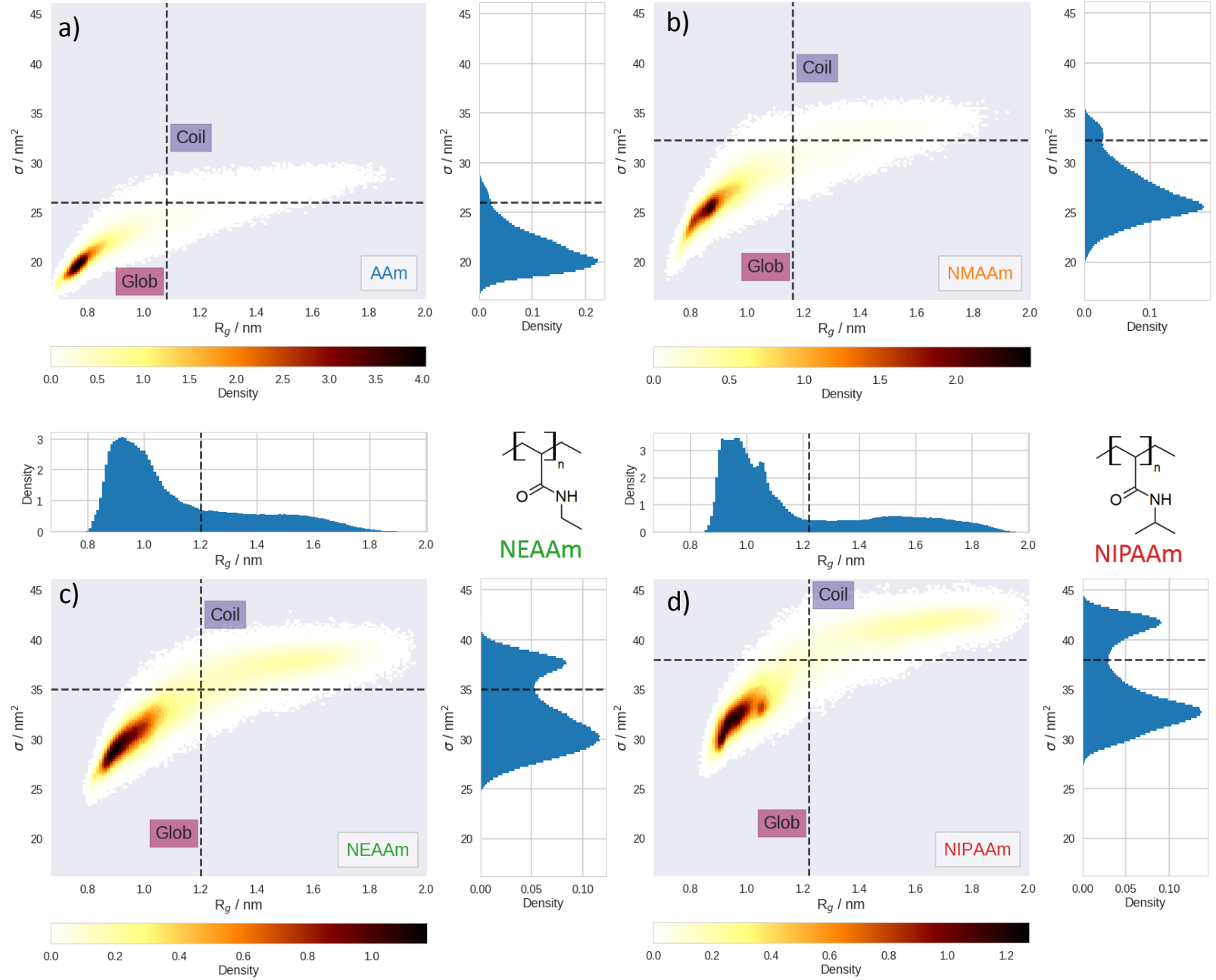

Figure S5 Two-dimensional histograms of polymer conformations in radius of gyration $\left(R_{g}\right)$ and solvent accessible surface area $(\sigma)$. The respective one-dimensional distribution of values is shown above and on the right, respectively. We mark the regions in the plane with C structures, with a purple label "Coil" and the regions with G, with a red label "Glob", respectively.

a) $A A m$, b) NMAAm, c) NEAAm, and d) NIPAAm.

Conformations in the upper left and the lower right quadrant of this two-dimensional plane have been discarded for the subsequent analysis, since their conformational state was not distinctly assigned by our method. The number of these conformations is generally small. 
State Assignation of All Conformations
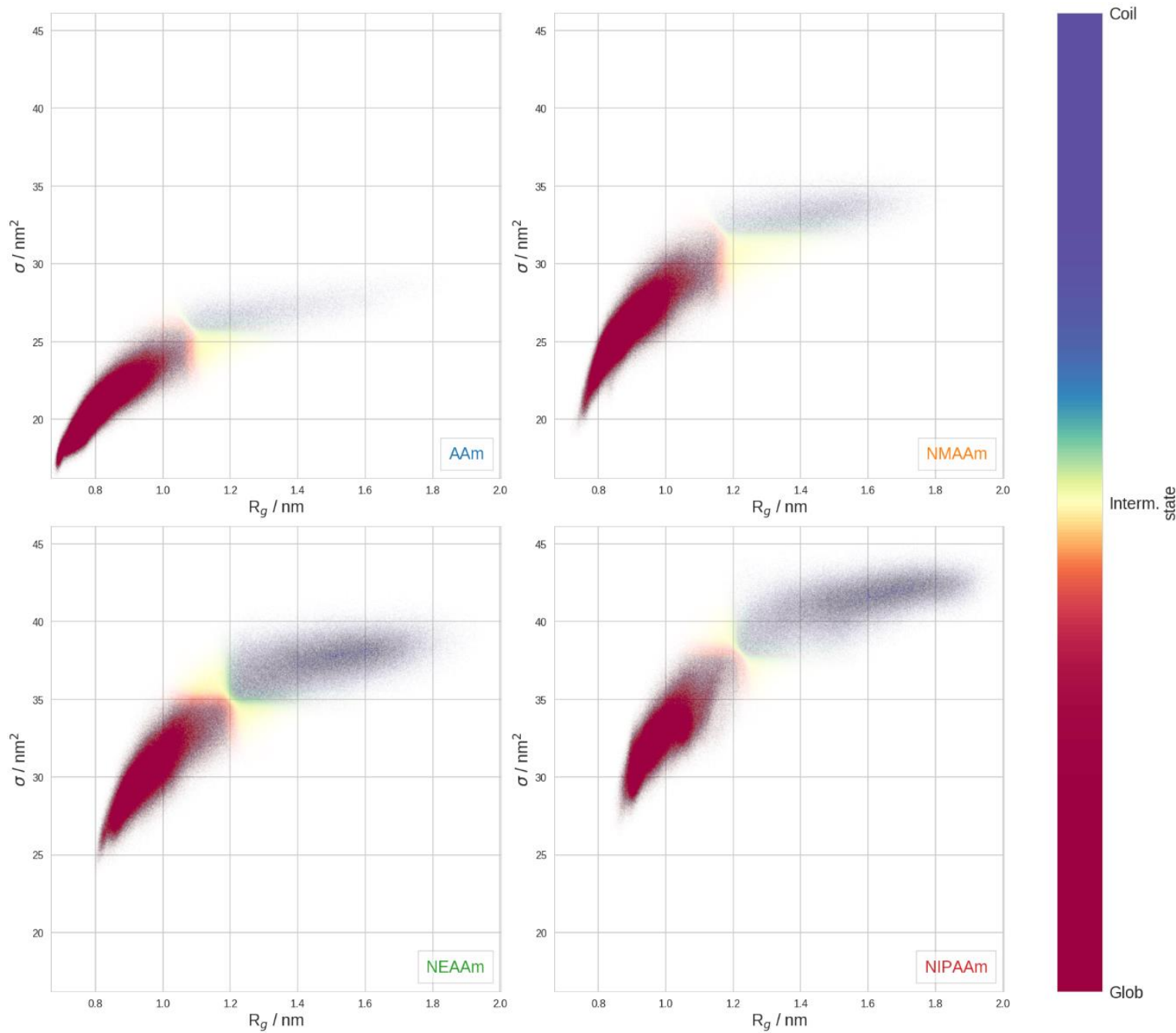

Figure S6 State definition in the two-dimensional radius of gyration $\left(R_{g}\right)$ and solvent accessible surface area $(\sigma)$ plane. Globular conformations are depicted in red, Coil conformations are depicted in violet between these states we model a smooth transition. 
State Borders and Means in Conformational States

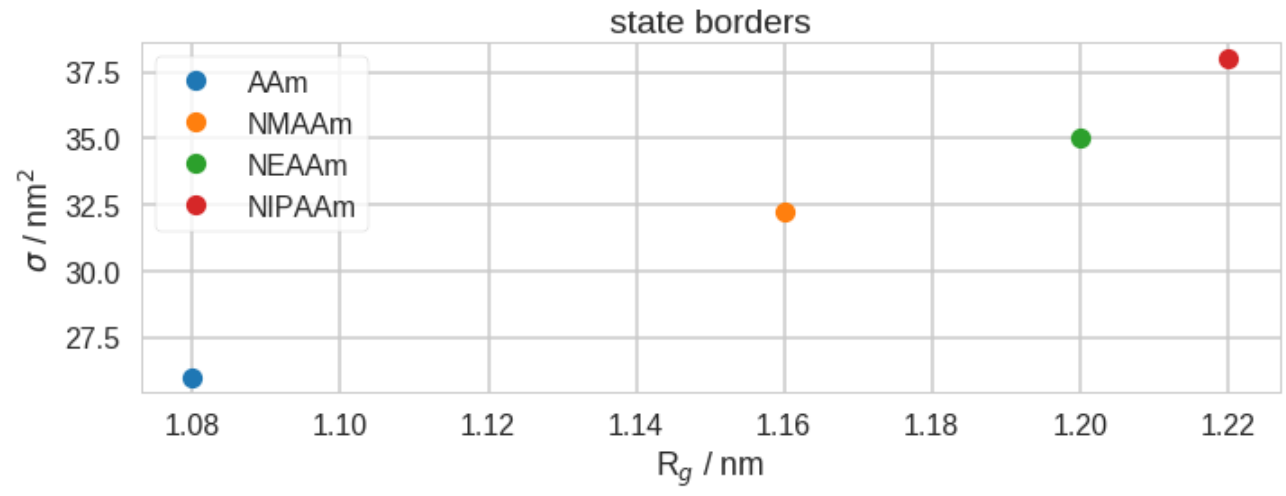

Figure S7 State borders between C and G for all polymers in $R_{g}-\sigma$ plane.
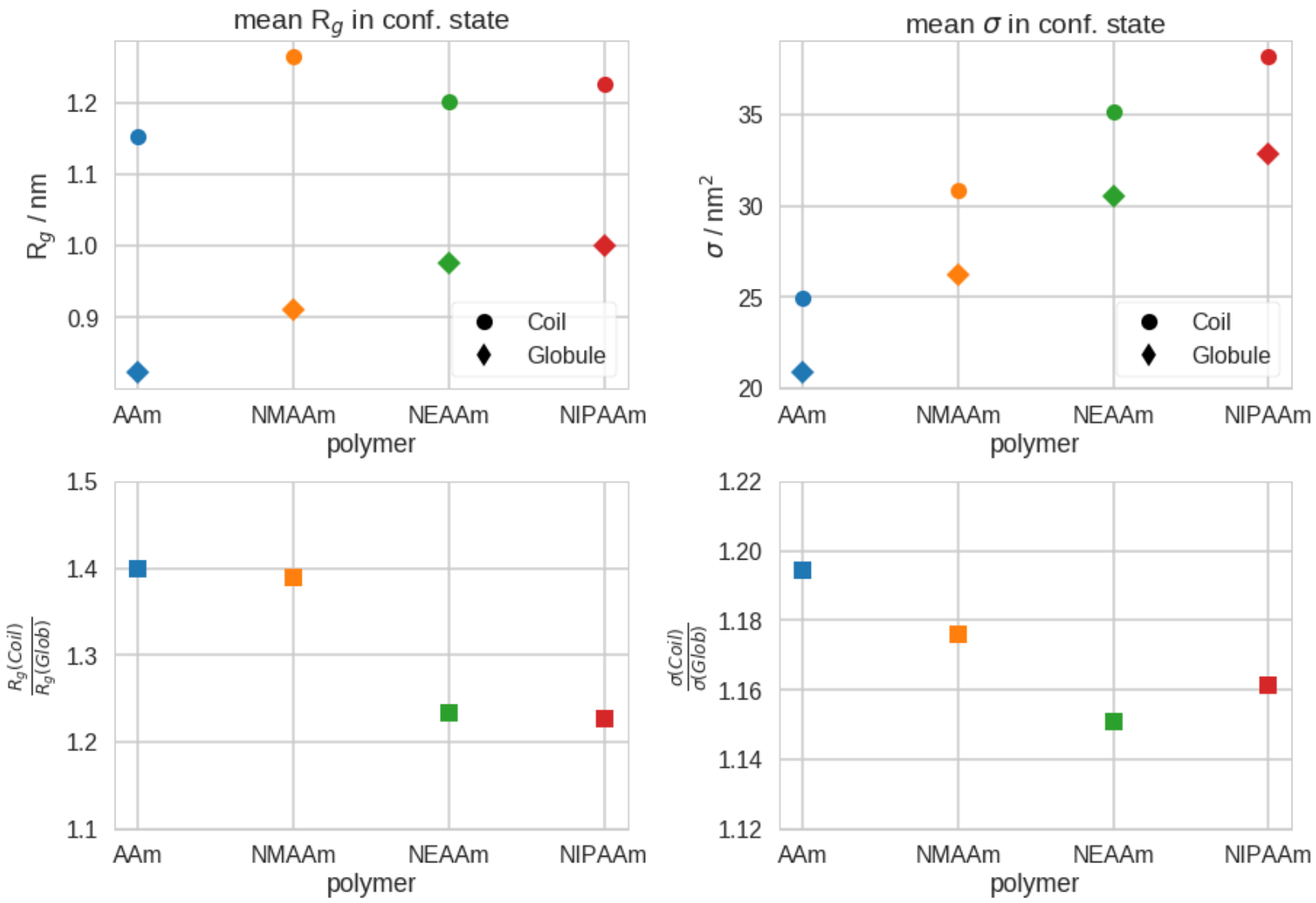

Figure S8 Mean $R_{g}$ and $\sigma$ in $C$ and $G$ for all polymers. In the left panels we show $R_{g}$ in the right panels we show $\sigma$. In the upper panels, we show mean values within the conformational ensembles, in the lower panel we show the quotient of the mean in the $C$ over the mean in the $G$. 
Ratio of Radius of Gyration and Hydrodynamic Radius

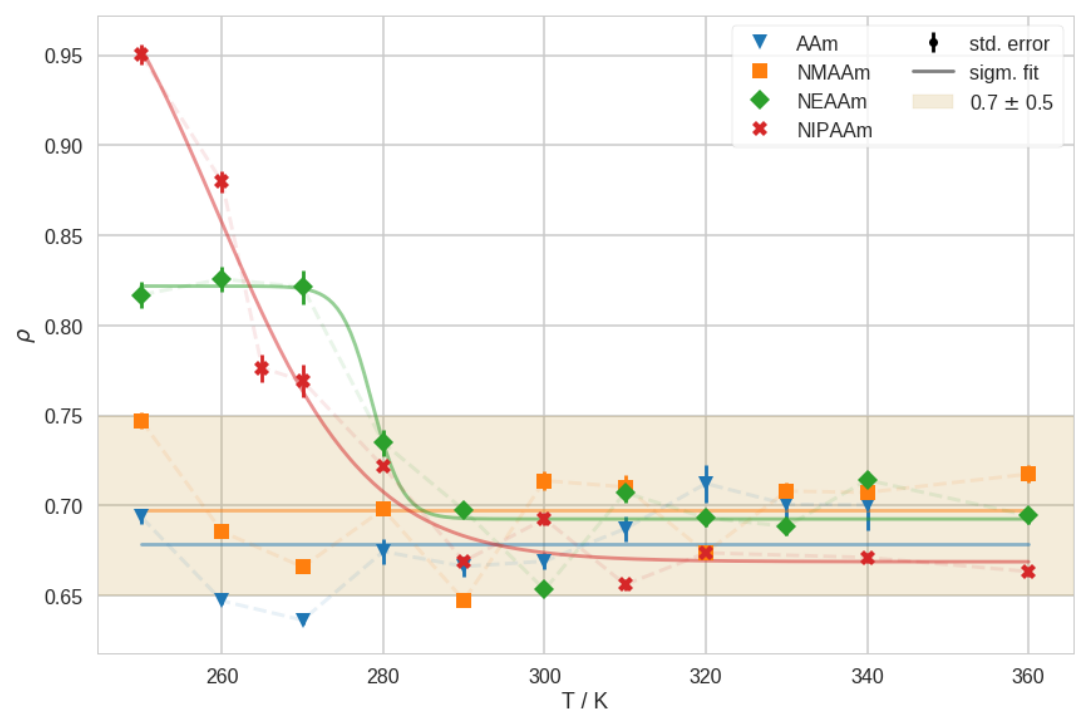

Figure S9 Ratio of radius of gyration over hydrodynamic radius, $\rho=R_{g} / R_{h}$. For the set of polymers, we calculate the arithmetic mean and the standard error of the mean of $\rho$ at the respective temperature. In blue triangles we show data points from simulations of AAm, in orange squares NMAAm, in green diamonds NEAAm and in red crosses NIPAAm. Where applicable we show the logistic fit of the data in the respective color. 


\section{Persistence Length}

In order to quantify the polymer stiffness, we modelled the polymer backbone using three dimensional cubic splines. Therefore, we performed the interpolation in the three Cartesian coordinate directions independently. This process has been implemented in Python, making use of the MDAnalysis, the SciPy and the csaps packages ${ }^{1-3}$. As a result, we obtained a smooth representation of the polymer backbone for every frame. With this representation, we calculated bending angles along the polymer backbone with respect to the polymer terminus. Averaging over the whole set of sampled structures at every temperature, we calculated the persistence length $\mathrm{P}$, as

$$
P=-\frac{L}{\ln (\langle\cos \theta\rangle)}
$$

Where $L$ is the distance along the backbone and $\langle\cos \theta\rangle$ is the mean cosine of the bending angle ${ }^{4}$.
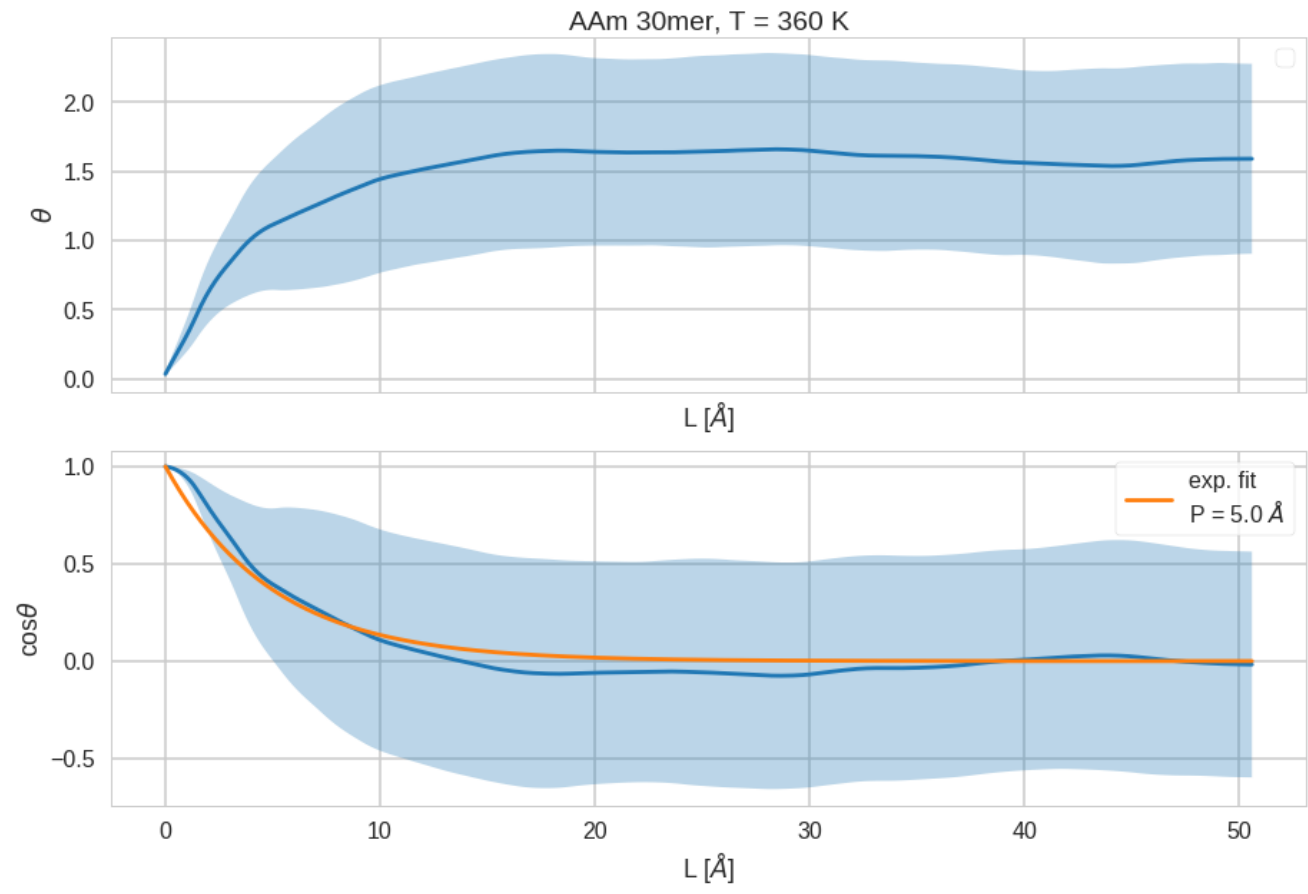

Figure 10 Exemplary depiction of the fit of the persistence length. Top panel: Mean bending angle at increasing distance on the polymer backbone of AAm at $360 \mathrm{~K}$ depicted as blue line, standard deviation is given in light blue. Bottom panel: mean cosine of the bending angle $\theta$ with exponential fit (orange line) of the progression of this curve. From this fit we obtain the persistence length according to Eq. S1. 
We determined the persistence length $(P)$ of the polymers at different temperatures, according to Eq. S1.

These results are depicted in SI-Figure S11. Generally, the persistence lengths of the set of polymers is similar within the range of variance. According to this simple analysis, we mostly obtain persistence lengths between 3 to $8 \AA$ A i.e., 2 to 4 monomer lengths. NIPAAm appears to be slightly less flexible than the rest of the set. In addition, NIPAAm's persistence length increases above the CGT temperature.
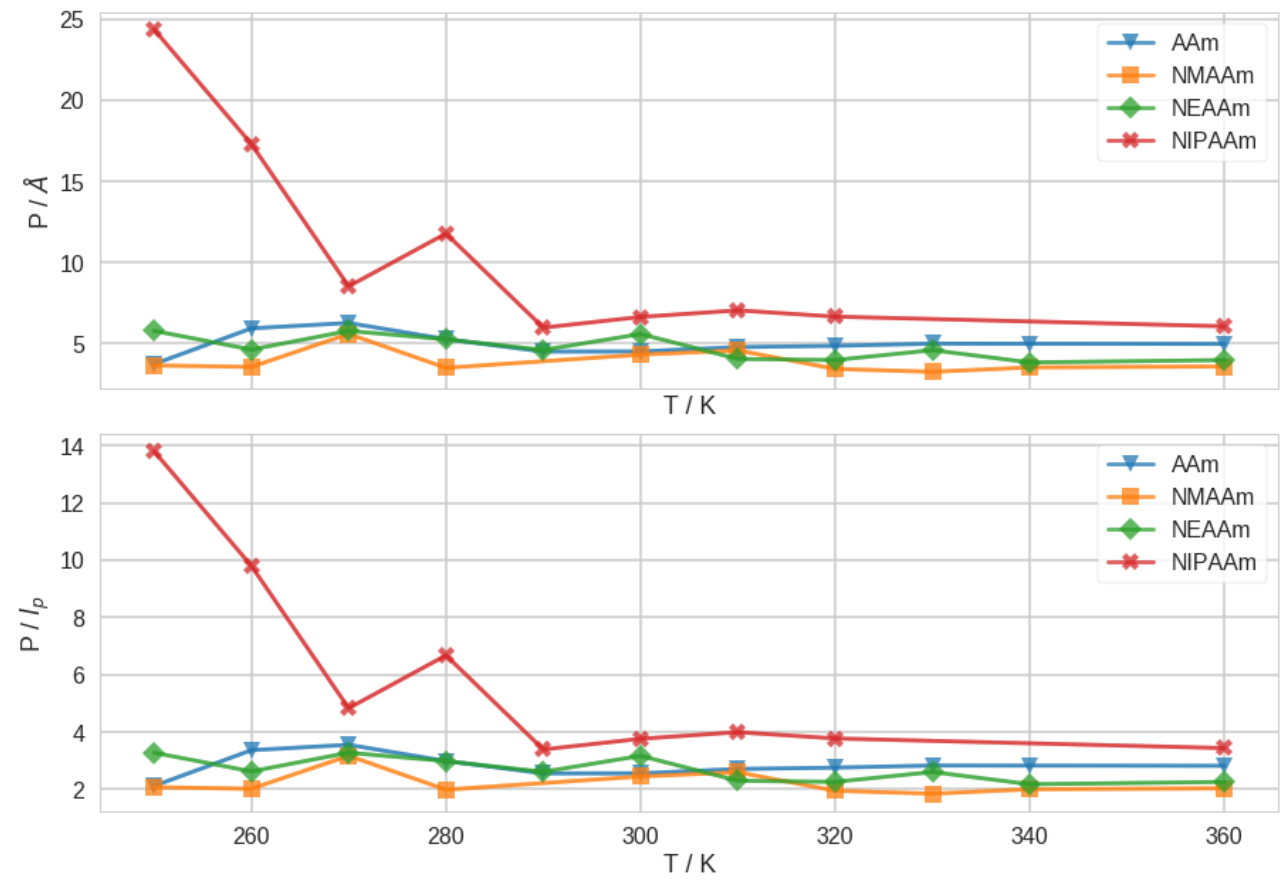

Figure S11 Persistence length $(P)$ of different polymers at different temperatures $(T)$. We calculated the persistence length of the different polymers from the $2 \mu \mathrm{s}$ simulations according to $\mathrm{Eq}$. S1 In blue triangles we show data points from simulations of AAm, in orange squares NMAAm, in green diamonds NEAAm and in red crosses NIPAAm. Both panels show the same data, but in different units. Top panel: $P$ in Ångstrom, bottom panel: $P$ in multiples of the monomer length. 
The persistence length is frequently used to compare the general stiffness of polymers ${ }^{5,6}$. Nevertheless, it is challenging to precisely estimate persistence lengths of linear polymer chains, experimentally. We were able to assess persistence lengths from our simulations, which agree with experimental values within the margin of variance. We believe, that sampling a large number of transitions improves the estimate of the persistence length, since averaging the bending of the polymer over a small number of $\mathrm{G}$ structures is not fully representative for the structural diversity sampled in experiments. Therefore, we are more confident in the estimates of the persistence length in the simulations at high temperatures. Above $270 \mathrm{~K}$, we report an average persistence lengths of NIPAAm of $P \approx 7.5 \pm 2 \AA$. Experimentally estimated persistence lengths of NIPAAm lie between approx. 2.5 and $7.5 \AA^{7,8}$ depending on solvent conditions. Concluding from that, we estimate the Kuhn length of this set of polymers, including NIPAAm to be between 5 and $15 \AA$, respectively approximately 4-7 monomers. 
Internal Hydrogen Bonds
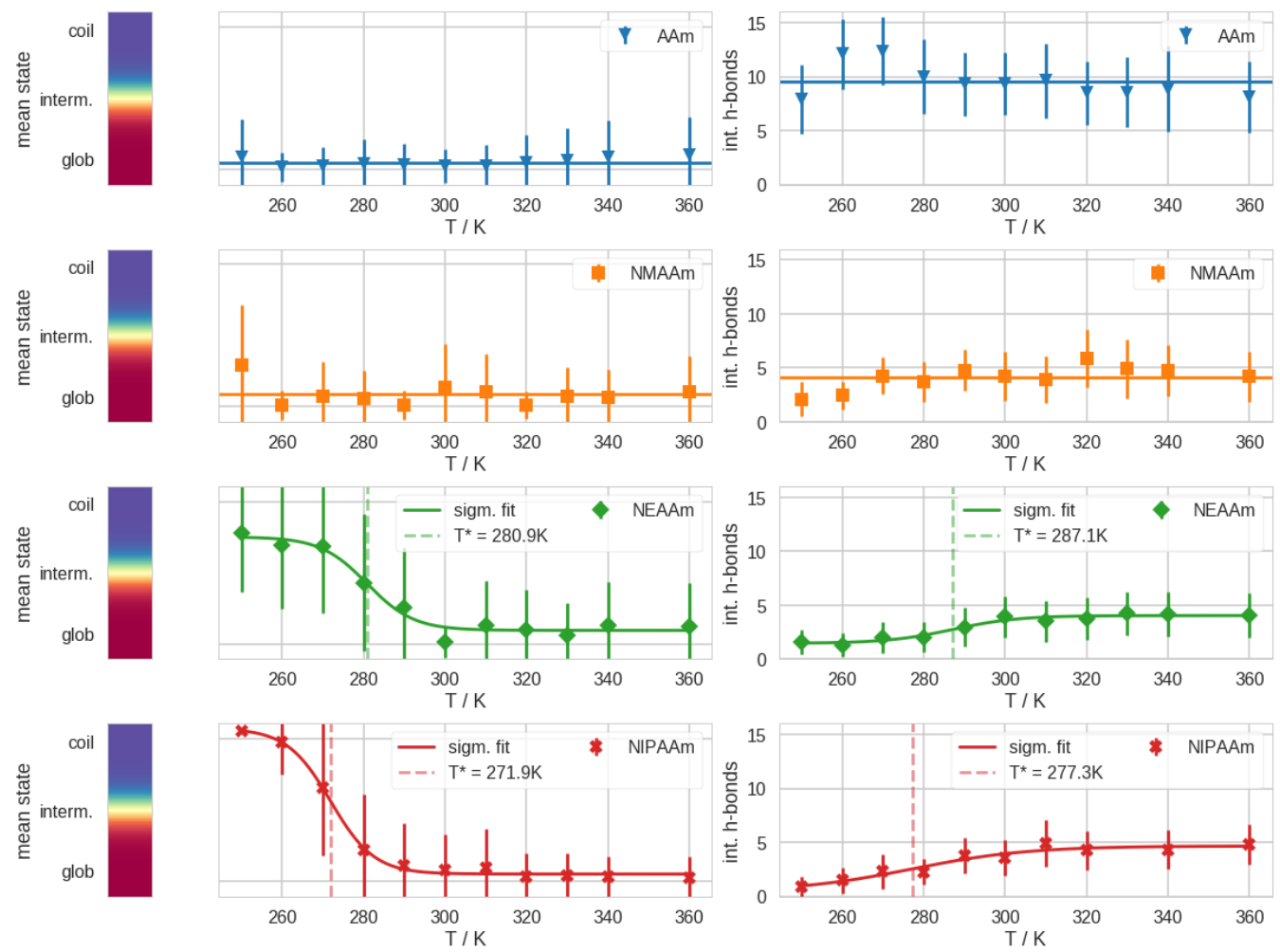

Figure S12 Comparison of mean number of internal hydrogen bonds with the mean conformational state. In the left panels we show the mean conformational state at increasing simulation temperatures. In the right panels we show the mean number of internal hydrogens bonds at different temperatures. Where applicable, we apply a logistic fit (orange). From top to bottom: AAm in blue triangles, NMAAm in orange squares, NEAAm in green diamonds, NIPAAm in red crosses. 

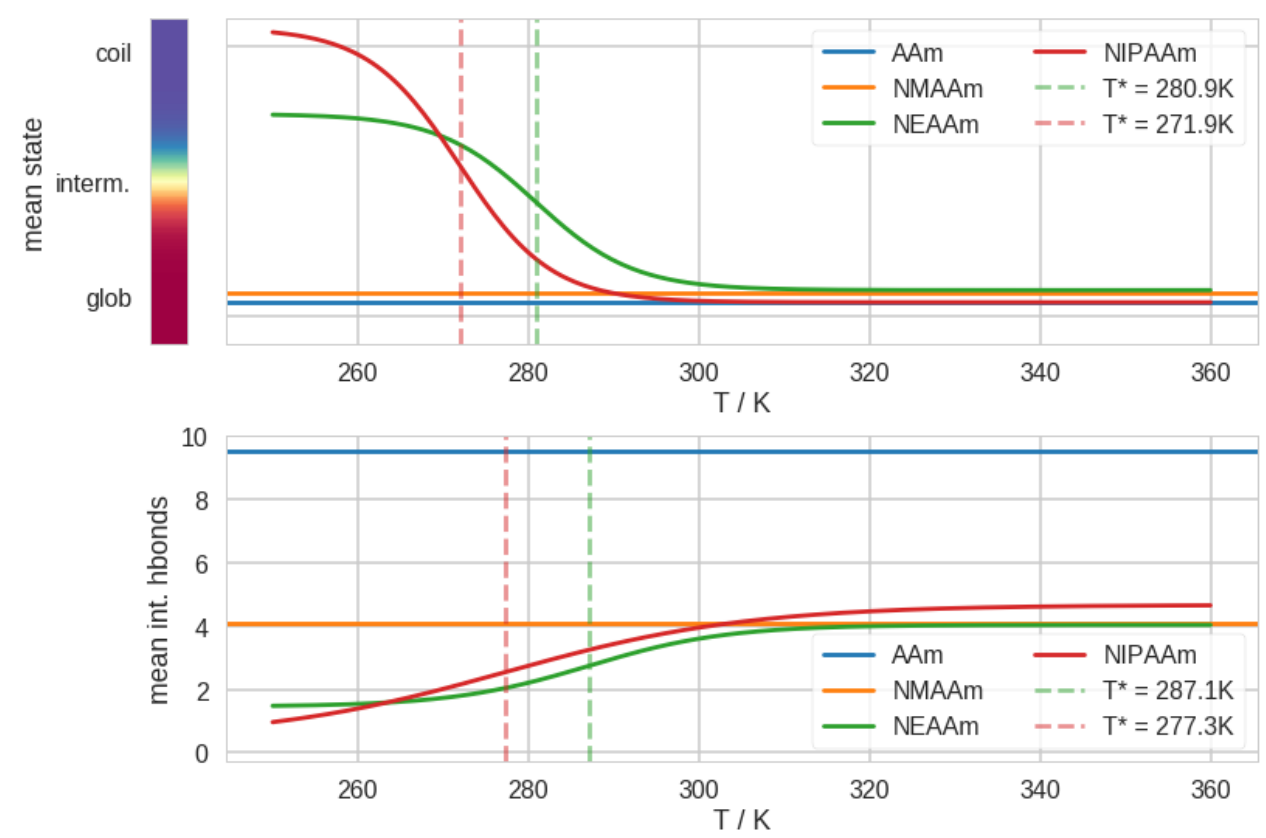

Figure S13 Top panel: interpolation of the mean conformational state at different temperatures. Bottom panel: interpolation of the mean number of internal hydrogen bonds at different temperatures. This figure summarizes Figure S12. In both panels we show AAm in blue, NMAAm in orange, NEAAm in green and NIPAAm in red. 
Naïve Mean of $v$ and $v_{\sigma}$ at Different Temperatures
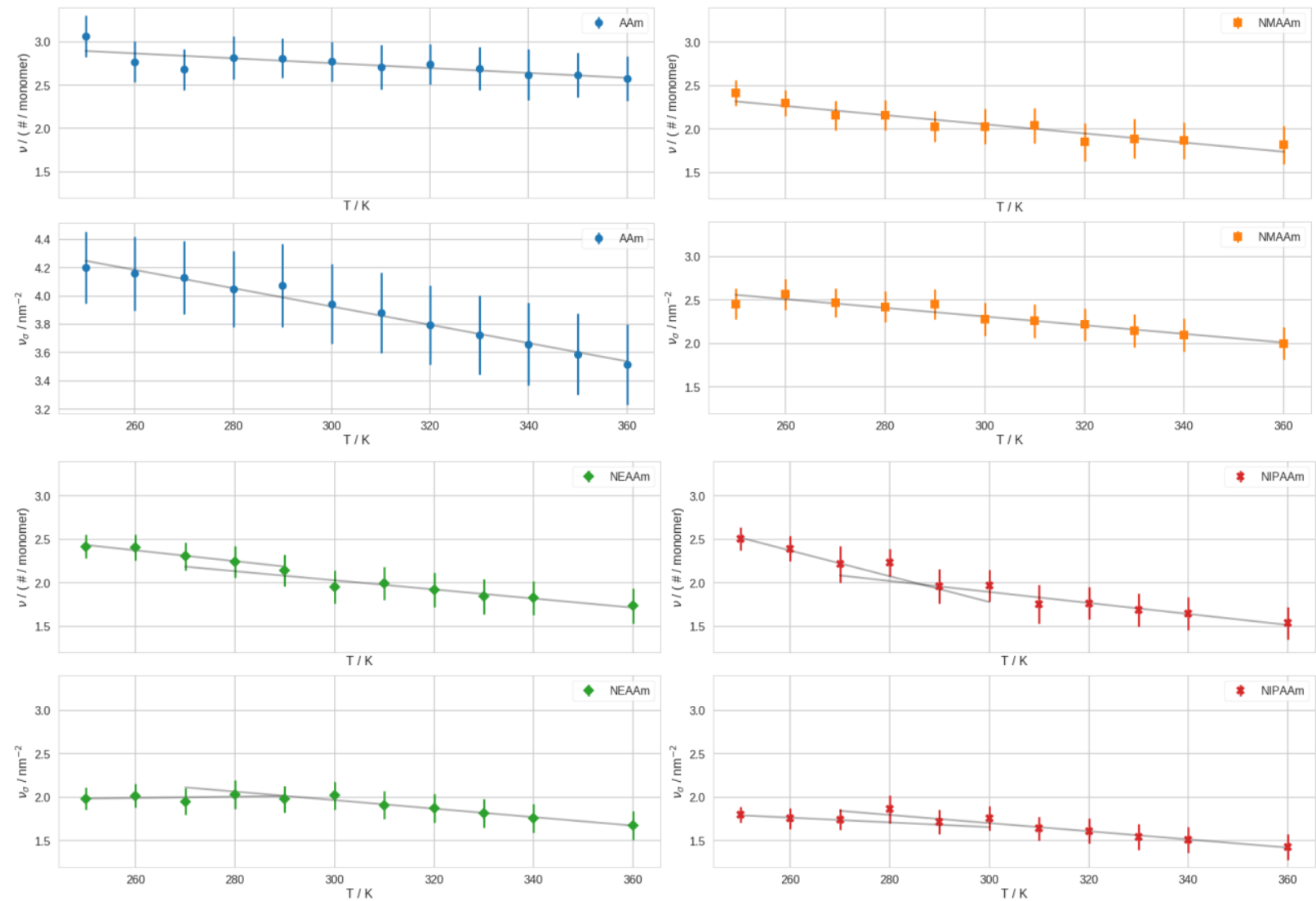

Figure S14 Naïve means of number of hydrogen bonds ( $v)$ and number of hydrogen bonds per solvent accessible surface area $\left(v_{\sigma}\right)$. a) AAm, b) NMAAm, c) NEAAm, d) NIPAAm. In the respective panels, we show $v$ in the upper axis and $v_{\sigma}$ in the lower axis. We calculate the naive mean and the standard deviation of these quantities and perform linear fit for these points. Please note the different $y$-axis scale for AAm. 
Hydrogen Bonds of Amid Group With Water
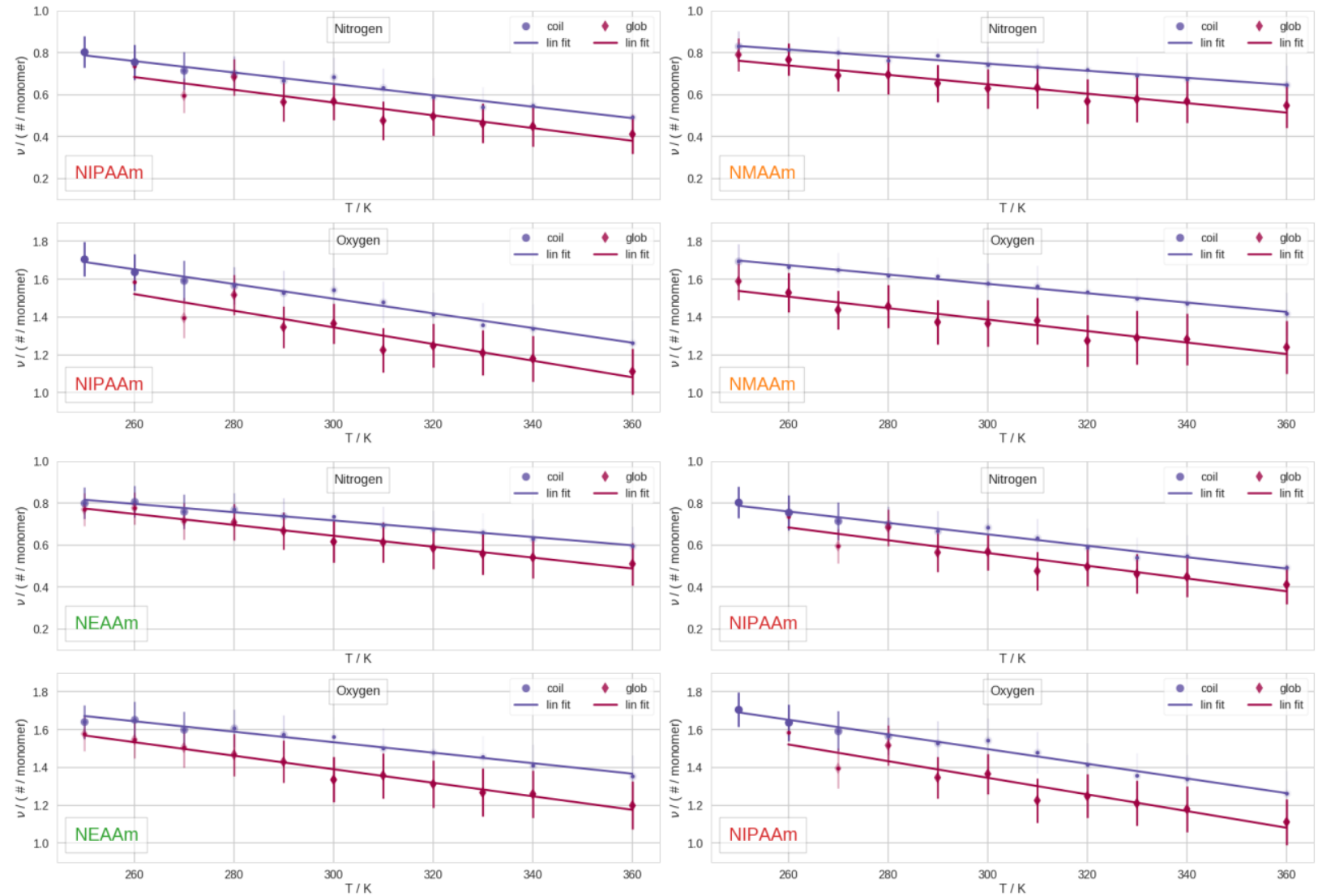

Figure S15 Mean number of hydrogen bonds between the solvent and the polymers at different temperatures within the different ensembles. The hydrogen bonds between water and the nitrogen, respectively the oxygen of the polymer are counted separately. The conformational subensembles of $C$ and $G$ are analyzed separately. We show results related to $C$ in purple spheres and results related to $G$ in dark red diamonds. 


\section{Differences in Enthalpy}
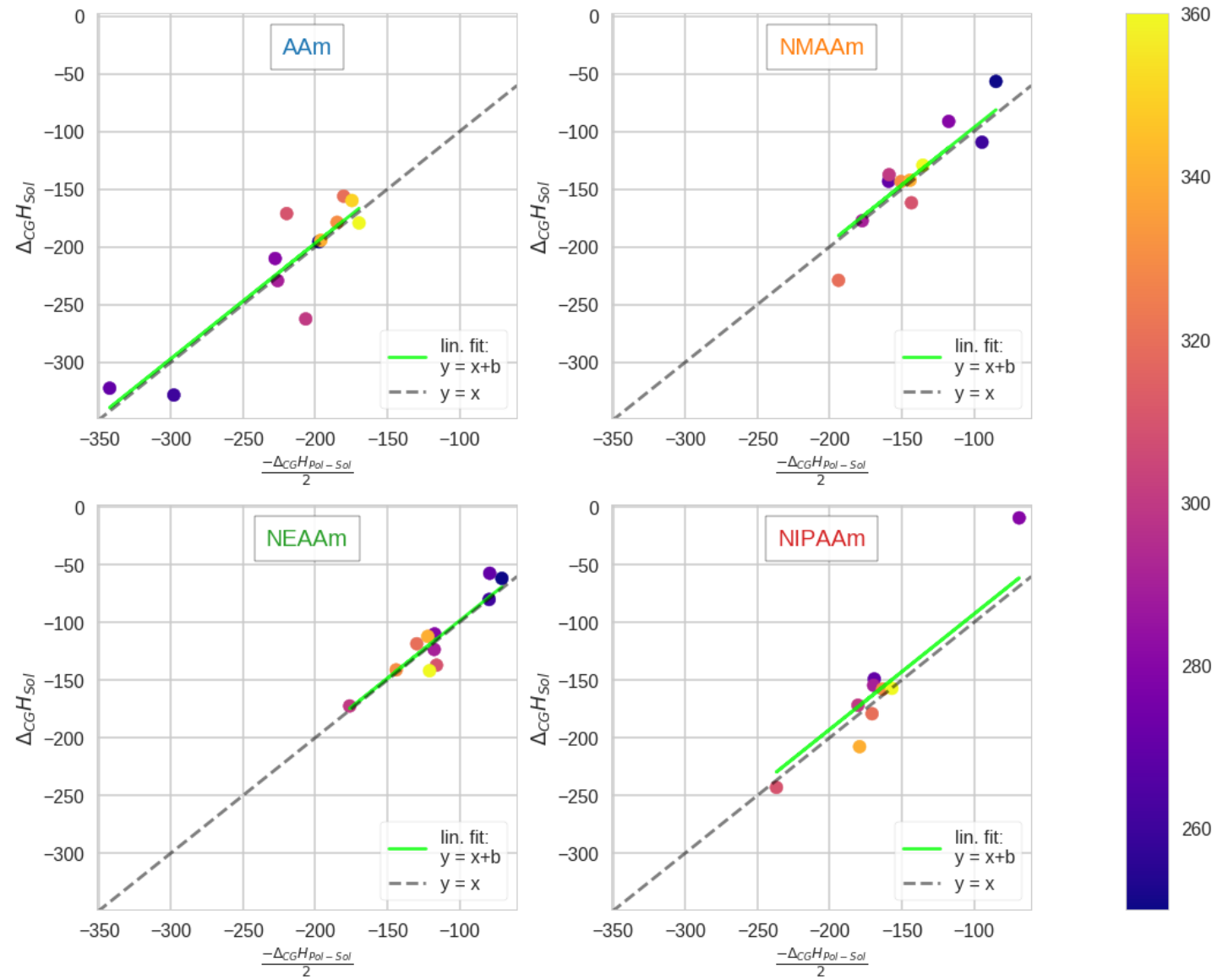

340

320

Figure S16 Comparison of enthalpic contributions with the CGT. $\Delta_{C G} H_{S o l}$ is the difference in internal energy of the solvent-solvent interactions, $\triangle_{C G} H_{S o l-P o l}$ is difference in internal energy of the polymer-solvent interactions. We show data related to the respective polymers as follows: (A) upper left: $A A m$; (B) upper right: NMAAm; (C) lower left: NEAAm and (D) lower right: NIPAAm. In all panels, we plot the mean difference of these two quantities at different temperatures. The corresponding simulation temperature is color coded in accordance to the color bar. In order to facilitate the comparison of the magnitude of these two properties, we plot $\mathrm{x}=\mathrm{y}$ as a dashed grey line. 

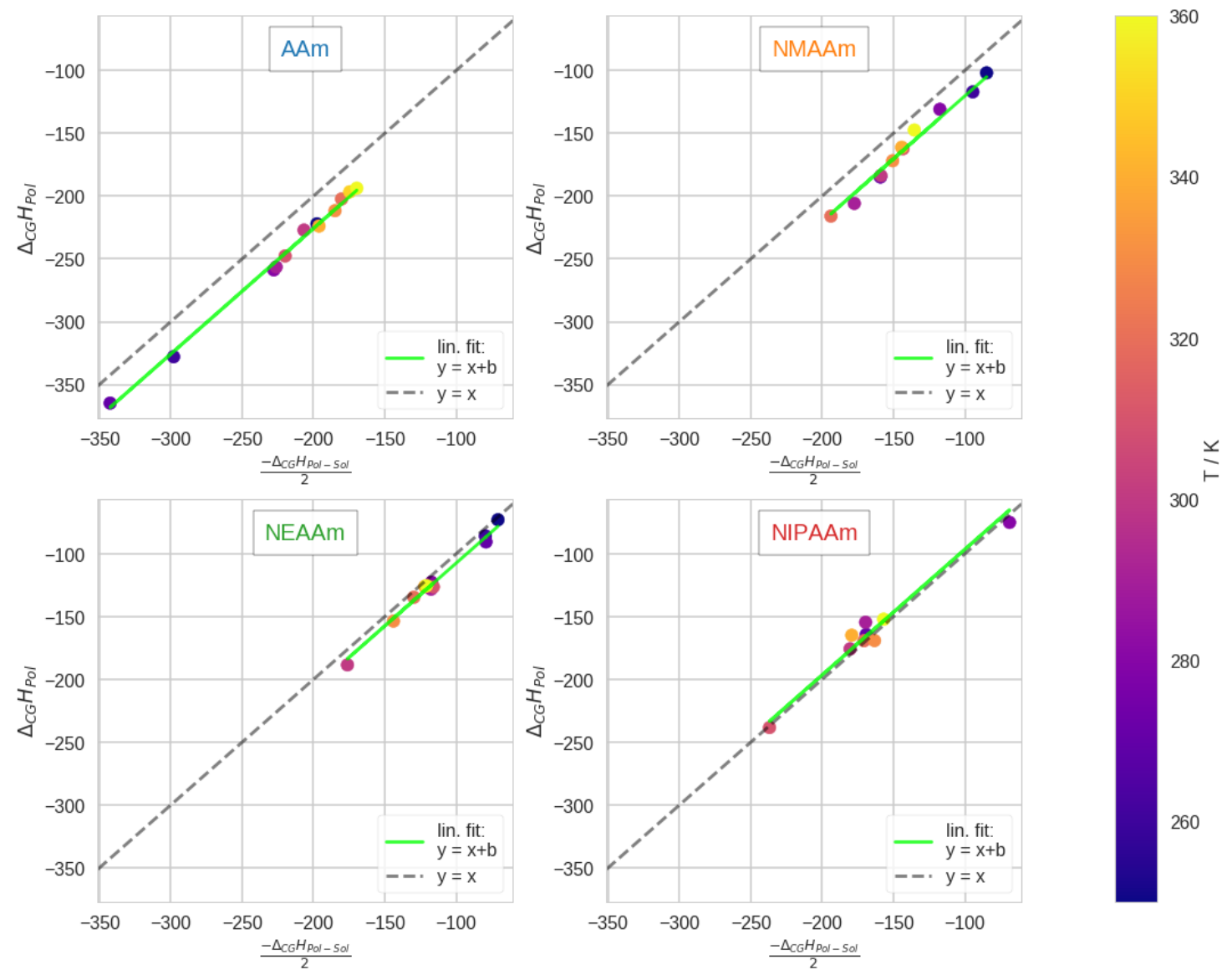

Figure S17 Comparison of enthalpic contributions with the CGT. $\Delta_{C G} H_{P o l}$ is the difference in internal energy of the polymer-polymer interactions, $\Delta_{C G} H_{S o l-P o l}$ is difference in internal energy of the polymer-solvent interactions. We show data related to the respective polymers as follows: $(A)$ upper left: $A A m$; $(B)$ upper right: NMAAm; (C) lower left: NEAAm and (D) lower right: NIPAAm. In all panels, we plot the mean difference of these two quantities at different temperatures. The corresponding simulation temperature is color coded in accordance to the color bar. In order to facilitate the comparison of the magnitude of these two properties, we plot $\mathrm{x}=\mathrm{y}$ as a dashed grey line. 
Since the potential energy is generally not a function of temperature, it is no surprise to not find a consistent trend of these values with the simulation temperature. Nevertheless, sampling a different conformational ensemble at different temperatures, may lead to different results for the mean potential energy at different temperatures.
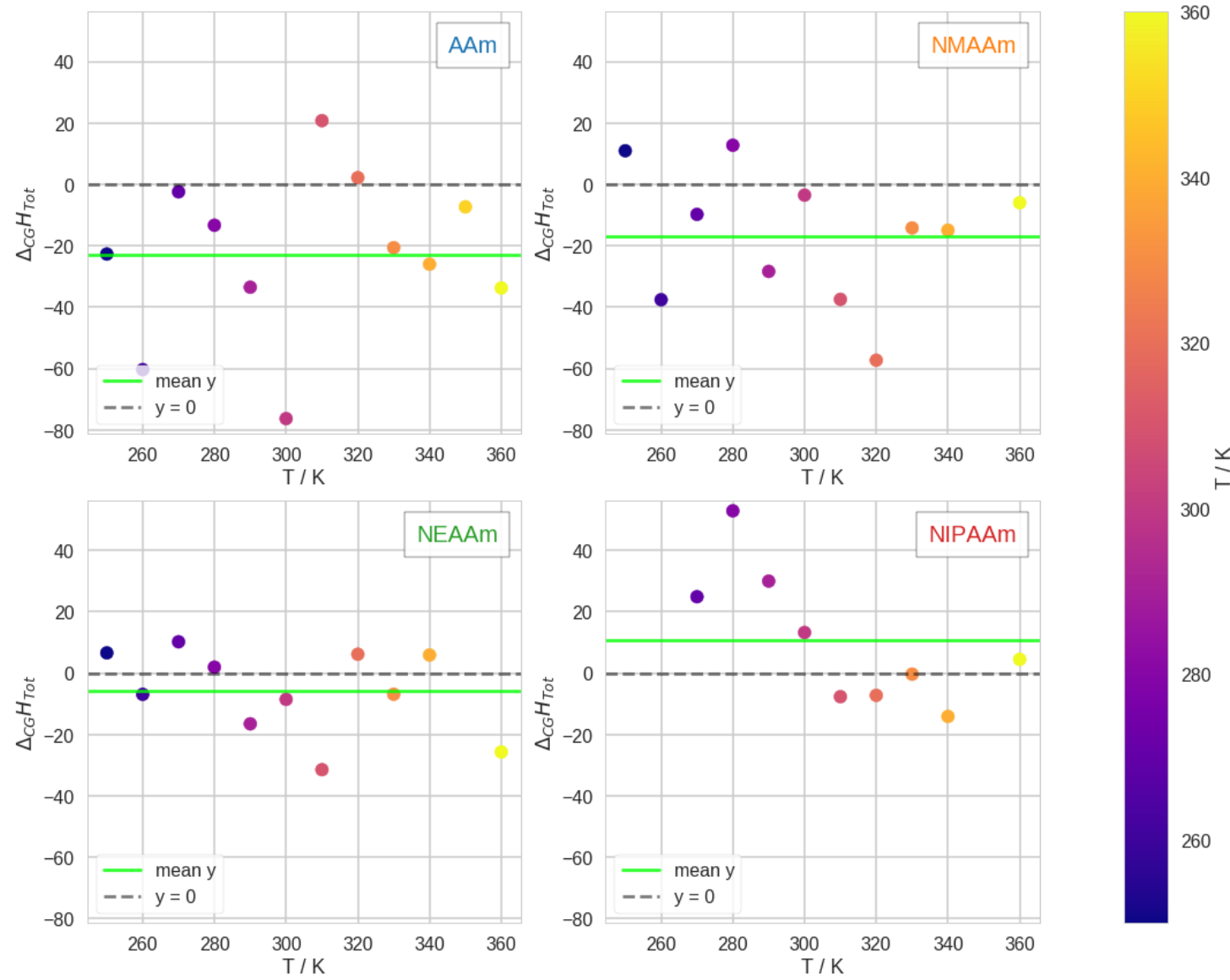

Figure S18 Mean difference in total Enthalpy with the CGT. 
Lifetimes of Hydrogen Bonds
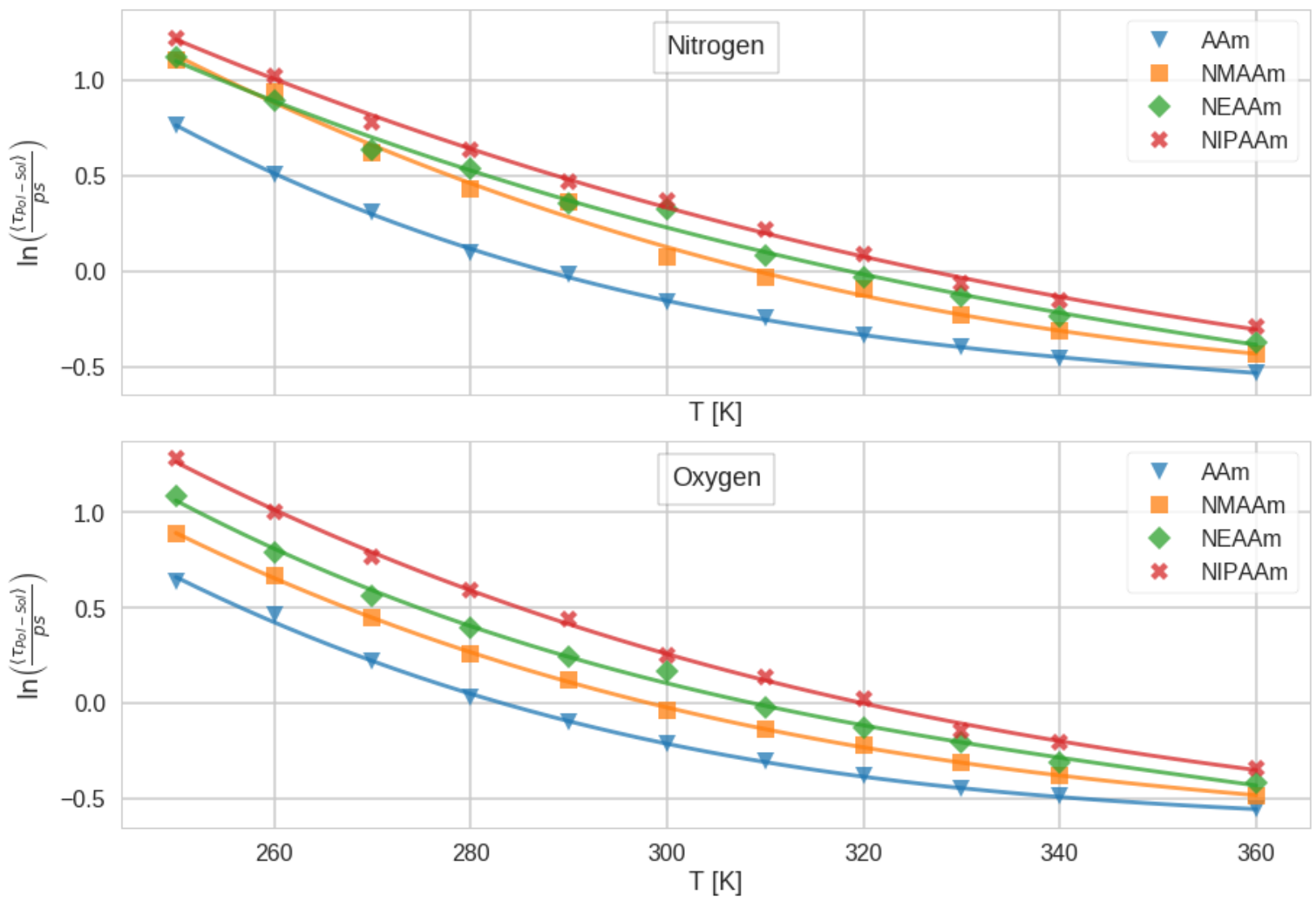

Figure S19 Mean lifetimes of hydrogen bonds between polymers and water. In blue, we show data points from simulations of $A A m$, in orange NMAAm, in green NEAAm and in red NIPAAm. 


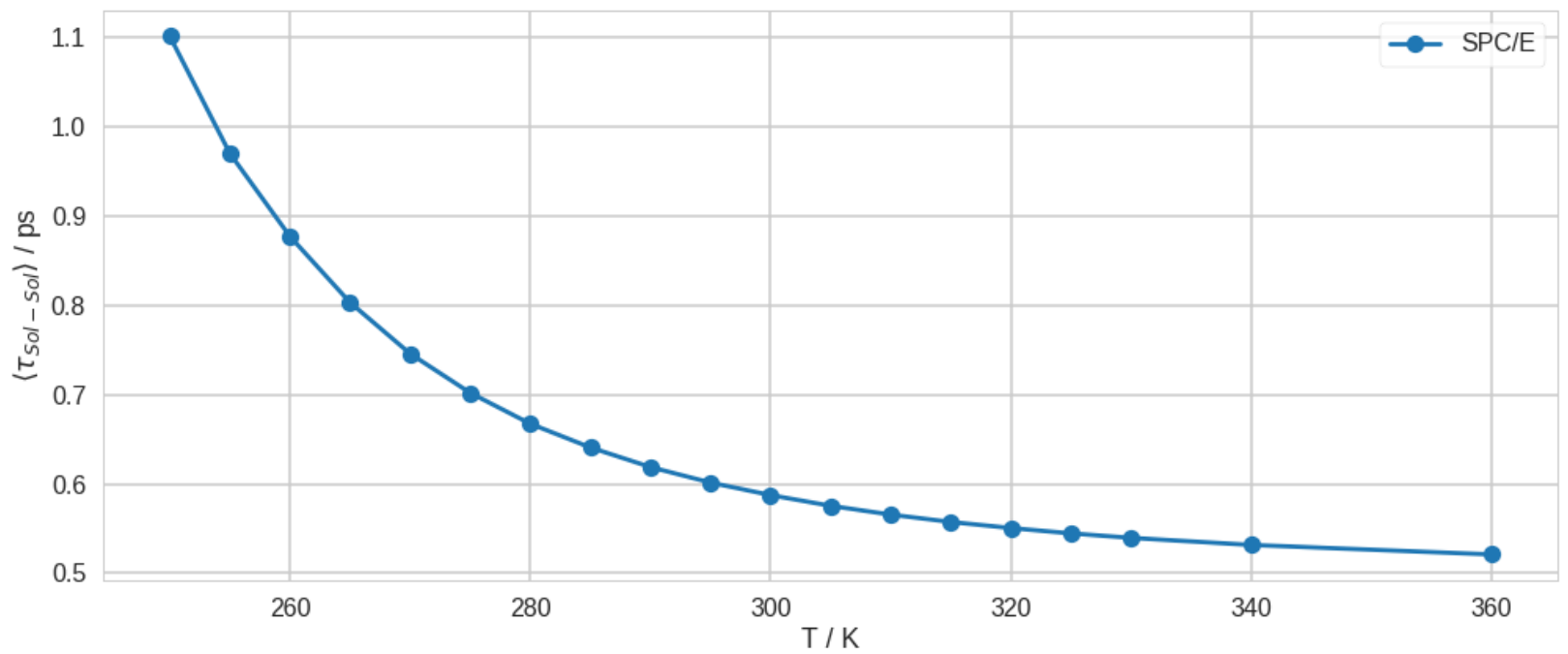

Figure S20 Mean lifetimes of hydrogen bonds in bulk solvent at different temperatures for SPC/E water model. 


\section{Force Field Parameters}

Since we expect the partial charges of the polymers to be of major importance for the thermosensitive behavior, we list these below, Table S1. We expect the bounded parameters, as well as the $\amalg$ parameters not to cause large deviations in thermosensitive behavior. Furthermore, we want to emphasize here again that we expect the water model to cause large shifts in the Coil-Globule transition temperature.

Table S1 Force field parameters for the set of polymers with the OPLS force-field ${ }^{9}$. Generated with the Maestro software package by Schroedinger ${ }^{10}$. We schematically explain the atom labels in Figure S21.

\begin{tabular}{|c|c|c|c|c|}
\hline Polymer & AAm & NMAA & NEAA & NIPA \\
\hline Atomtype & Charge / e & & & \\
\hline $\mathrm{C}^{\mathrm{BT} 1}$ & -0.18 & -0.18 & -0.18 & -0.18 \\
\hline$C^{B 1}$ & -0.06 & -0.06 & -0.06 & -0.06 \\
\hline $\mathrm{C}^{\mathrm{B} 2}$ & -0.12 & -0.12 & -0.12 & -0.12 \\
\hline $\mathrm{C}^{\mathrm{BT} 2}$ & -0.12 & -0.12 & -0.12 & -0.12 \\
\hline$C^{A}$ & 0.5 & 0.5 & 0.5 & 0.5 \\
\hline 0 & -0.5 & -0.5 & -0.5 & -0.5 \\
\hline$N$ & -0.76 & -0.5 & -0.5 & -0.5 \\
\hline$C^{S P}$ & & & 0.08 & 0.14 \\
\hline$C^{S T}$ & & -0.18 & -0.18 & -0.18 \\
\hline $\mathrm{H}^{\mathrm{C}}$ & 0.06 & 0.06 & 0.06 & 0.06 \\
\hline $\mathrm{H}^{\mathrm{N}}$ & 0.38 & 0.3 & 0.3 & 0.3 \\
\hline
\end{tabular}




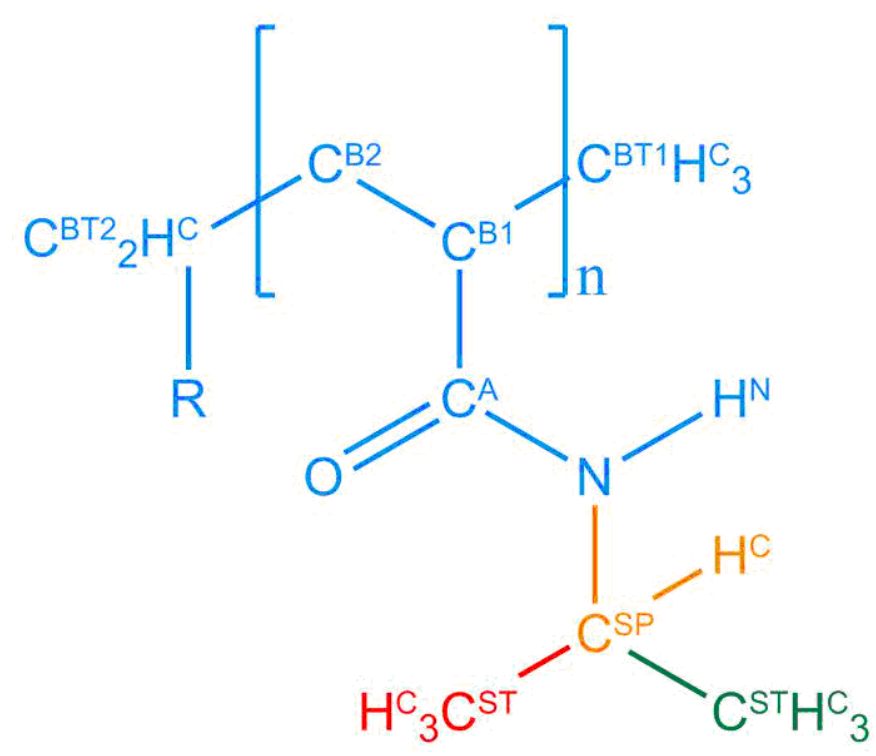

Figure S21 Annotation of atom types in polymer chains. We show an annotated monomer unit of NIPAAm. Superscript annotations refer to the following: $B \rightarrow$ polymer backbone; $A \rightarrow$ amide; $S P \rightarrow$ substituent proximal; $S T \rightarrow$ substituent terminal.

Furthermore, BT1 and BT2 refer to the terminal ends of the polymer backbone. In Blue we show all atoms types, which exist in AAm. Orange, green and red color shall visualize, which atoms are added for NMAAm, NEAAm and NIPAAm respectively. Not all hydrogen atoms at the $C$ atoms of the backbone are explicitly shown here. 


\section{References}

(1) Gowers, R.; Linke, M.; Barnoud, J.; Reddy, T.; Melo, M.; Seyler, S.; Domański, J.; Dotson, D.; Buchoux, S.; Kenney, I.; Beckstein, O. MDAnalysis: A Python Package for the Rapid Analysis of Molecular Dynamics Simulations; 2016; pp 98-105.

(2) Virtanen, P.; Gommers, R.; Oliphant, T. E.; Haberland, M.; Reddy, T.; Cournapeau, D.; Burovski, E.; Peterson, P.; Weckesser, W.; Bright, J.; van der Walt, S. J.; Brett, M.; Wilson, J.; Millman, K. J.; Mayorov, N.; Nelson, A. R. J.; Jones, E.; Kern, R.; Larson, E.; Carey, C. J.; Polat, I.; Feng, Y.; Moore, E. W.; VanderPlas, J.; Laxalde, D.; Perktold, J.; Cimrman, R.; Henriksen, I.; Quintero, E. A.; Harris, C. R.; Archibald, A. M.; Ribeiro, A. H.; Pedregosa, F.; van Mulbregt, P. SciPy 1.0: Fundamental Algorithms for Scientific Computing in Python. Nat. Methods 2020, 17, 261-272.

(3) Prilepin, E. CSAPS.

(4) Feldman, D. The Theory of Polymer Dynamics, by M. Doi and S. F. Edwards, the Clarendon Press, Oxford University Press, New York, 1986, 391 Pp. Price: \$78.50. J. Polym. Sci. Part C Polym. Lett. $1989,27,239-240$.

(5) Ito, D.; Kubota, K. Solution Properties and Thermal Behavior of Poly( N - n -Propylacrylamide) in Water. Macromolecules 1997, 30, 7828-7834.

(6) Takahashi, R.; Tokunou, H.; Kubota, K.; Ogawa, E.; Oida, T.; Kawase, T.; Nishinari, K. Solution Properties of Gellan Gum: Change in Chain Stiffness between Single- and Double-Stranded Chains. Biomacromolecules 2004, 5, 516-523.

(7) Kutnyanszky, E.; Embrechts, A.; Hempenius, M. A.; Vancso, G. J. Is There a Molecular Signature of the LCST of Single PNIPAM Chains as Measured by AFM Force Spectroscopy? Chem. Phys. Lett. 2012, 535, 126-130.

(8) Liang, X.; Nakajima, K. Nanofishing of a Single Polymer Chain: Temperature-Induced Coil-Globule Transition of Poly( N -Isopropylacrylamide) Chain in Water. Macromol. Chem. Phys. 2018, 219, 1700394.

(9) Jorgensen, W. L.; Maxwell, D. S.; Tirado-Rives, J. Development and Testing of the OPLS All-Atom Force Field on Conformational Energetics and Properties of Organic Liquids. J. Am. Chem. Soc. 1996, 118, 11225-11236.

(10) Schrödinger Release 2018-1: Maestro. Schrödinger, LLC: New York, NY 2018. 